\title{
Using mesh adaption for the identification of a spatial field of material properties
}

\author{
G. Puel*and D. Aubry \\ Laboratoire MSSMat (Ecole Centrale Paris/CNRS UMR 8579), Grande Voie des Vignes, 92290 Châtenay-Malabry, France
}

\begin{abstract}
SUMMARY
It is well known that the solution of an inverse problem is ill-posed and not unique. To avoid difficulties caused by this, when solving such a problem, Tikhonov's regularization terms are usually added to the norm quantifying the discrepancy between the model's predictions and experimental data. This regularization term however is often inadequate to perform the identification of a field of material properties which varies spatially. This is all the more difficult when dealing with the numerical solution of this inverse problem, for the sought field is spatially discretized and this discretization can influence the result of the identification.

We will here examine an overall strategy using classical adaptive meshing methods used to circumvent these drawbacks. The first step consists in using two distinct meshes: one associated with the discretization of the sought spatial field, the other associated with the solution of the mechanical problems (forward and adjoint states). In a second step, we will introduce local error estimators which allow an oriented refinement of the mesh associated with the sought parameters.

This general strategy is applied to a practical case study: the detection of underground cavities using experimental data obtained by an interferometric device on a satellite. We will then address the question of how the regularization terms and the error estimator driving the mesh refinement were selected. Copyright (c) 2010 John Wiley \& Sons, Ltd.

KEY WORDS: identification; inverse problem; adjoint state; regularization; mesh adaption; error estimators; quantity of interest.
\end{abstract}

\section{INTRODUCTION}

How should the material parameters of a domain be identified when the only available experimental data are measured on its boundaries? The solution of this inverse problem is well known for being ill-posed and not unique, so it becomes all the more challenging when the parameters to be identified depend on the space variable within the studied domain, and when the usually available measurements are scarce. Also, when solving this inverse problem numerically, the spatial discretization of the sought parameters field influences the whole identification process. As a result, the addition of relevant regularization terms to the inverse problem can be quite awkward.

This paper is focused specifically on the detection of underground cavities using experimental data obtained by an interferometric satellitar device. Techniques for the geometrical reconstruction

*Correspondence to: guillaume.puel@ecp.fr

Copyright (C) 2010 John Wiley \& Sons, Ltd. 
of buried objects (e.g. cracks, cavities or inclusions) through mechanical measurements have been largely investigated, as listed in the survey paper [1] dealing with inverse problems in elasticity. They generally consist in the minimization of a misfit function expressing the discrepancy between the available experimental data and the corresponding quantities calculated with the parameters field being identified. As regards the case of cavity detection, the minimization can be achieved through gradientbased optimization techniques used in conjunction with shape sensitivity formulations [2, 3]. Another approach consists in applying level sets methods to describe the shape of the sought cavity [4]. Instead of looking for the cavity's shape, it is also possible to try to directly identify the Young's modulus spatial field within the studied domain, as it is applied in specific examples in [5] or [6] using a specific constitutive relation error in addition to the initial misfit function.

In all cases, since theoretical studies have shown the complexity of this kind of inverse problems (see e.g. [7, 8] for considerations on uniqueness), prior knowledge is often required in order to avoid local minima For example, the shape of the cavity can be assumed, or the spatial Young's modulus can be sought as a piecewise constant field in a fixed spatial discretization. Techniques such as the linear sampling method $[9,10]$ or the topological derivative [11] also allow a preliminary probing of the medium and can provide an interesting initial guess. In a general way, however, regularization can be difficult to set or adapt.

The problem we address here leads to the same observations: when expressing this cavities detection issue in terms of an identification problem (the determination of the inner Young's modulus spatial field using the measured displacements on the surface of the studied ground), we stress the shortcomings of classical regularization such as Tikhonov's when the goal is to identify a spatially-variable field of material properties. Instead of assuming an arbitrary spatial discretization of the sought Young's modulus field, we introduce a strategy using the Finite Element Method (FEM) with a classical adaptive meshing in order to efficiently regularize the inverse problem.

Adaptive Finite Element techniques are only slowly considered for the solution of inverse problems $[12,13]$ and to our knowledge only $[14,15]$ and $[16,17]$ actually propose a specific method for solving inverse problems based on adaptive Finite Element meshes, which we want to adapt here in the case of elastostatics Partial Differential Equations (PDEs). The first step consists in using two distinct meshes: one associated with the discretization of the spatial field to be identified (e.g. Young's modulus), typically coarse and which will be progressively refined with adaption techniques, and one associated with the solution of the usual mechanical problems (e.g. stress-strain forward and adjoint state problems). It is shown that the choice of a coarse mesh to discretize the sought spatial field allows a good regularization of the problem, even if the identified parameters field is very roughly described. Therefore in a second step, the introduction of local error estimators can drive the refinement of the coarse mesh, resulting in an accurate identification of the sought spatial field, as it is demonstrated here in a practical case of study for an elastostatics problem.

\section{PRESENTATION OF THE PROBLEM}

Below are described the main features of the specific problem we want to address, which deals with the detection of underground cavities through measurements of the displacements at the free surface of the studied ground. This specific problem is used as an illustrational example of what can be obtained with the general strategy of identification we present here. 


\subsection{Experimental data}

From a practical point of view, experimental data come from a satellite equipped with an interferometric radar device measuring the displacement of given points of the surface of the ground, between two consecutive acquisitions, with an accuracy up to the millimeter, both in horizontal and vertical directions [18]. Such an experimental technique, called inSAR (synthetic aperture radar interferometry), allows the monitoring of a very fine grid of points, typically with a resolution of a dozen of meters, for areas up to a few hundreds of square kilometers. The idea is to use such a device to monitor the creation and/or the growth of underground cavities such as in [19], which presents the monitoring of the ground deformation of a site corresponding to the exploitation of underground salt by solution mining. For the problem addressed here, we assume that no cavity was present when the first acquisition was done, so the displacement $\Delta \underline{u}_{\exp }$ measured on the surface $\Sigma_{\exp }$ of the ground results from the creation of one or several cavities between the two consecutive acquisitions. Owing to the solution of the measurement process, $\Delta \underline{u}_{\text {exp }}$ will be considered as a continuous $3 \mathrm{D}$-vectorial field.

The considered detection problem then consists in finding the created cavities within a specific volumic domain that is characterized by the monitored surface and a given depth, which are set a priori. In the following, this domain is typically five kilometers deep and ten kilometers large, and the cavities that are to be found are several hundreds of meters large and several kilometers below the surface of the ground, which consequently drops locally a few tens of centimeters. This problem is depicted in a 2D schematic representation in Figure 1, where the monitored surface $\Sigma_{\exp }$ is symbolized with hatching.

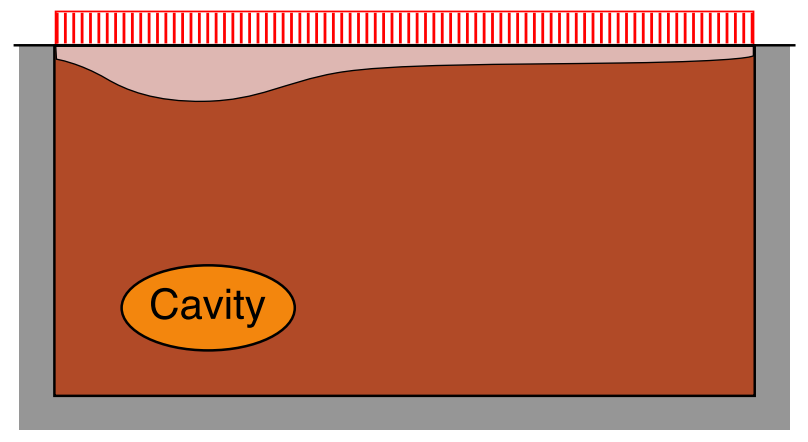

Figure 1. 2D schematic representation of the considered problem

\subsection{The forward model}

The forward model to be compared with experimental data relies on some simple non-restrictive assumptions. The initial ground (i.e. with no cavity) is considered as a homogeneous, isotropic medium, whose mechanical properties (Young's modulus $E_{0}$, Poisson's ratio $\nu_{0}$, mass density $\rho_{0}$ ) is well known. This description will be used as an initial guess in the identification process of the Young's modulus spatial field, as well as a means to know the initial depression of the surface of the ground due to gravity when no cavity is present. This depression is added to the displacement $\Delta \underline{u}_{e x p}$ measured between two acquisitions in order to obtain the whole displacement $\underline{u}_{\exp }$ of the surface of the ground, which is the result of the combined effects of gravity and of the creation of one or several cavities. As a conclusion, 
this displacement field $\underline{u}_{e x p}$ so defined is considered as the experimental data used in the identification process defined in the following.

The domain associated with the ground is denoted $\Omega$ and the following conditions are verified on the boundary $\partial \Omega$ of the domain $\Omega$ : whereas no load is applied on the surface $\Sigma_{\text {exp }}$ of the ground, we assume that the displacements on the others boundaries of the domain vanish. So the forward problem is defined as follows: given a Young's modulus spatial field $E$, we compute the displacement field $\underline{u}(E)$ in $\Omega$ with the following PDE and boundary conditions:

$$
\begin{aligned}
\underline{\operatorname{div}} E C_{0} \varepsilon(\underline{u})-\rho g \underline{e}_{z} & =\underline{0} & & \text { in } \Omega \\
\underline{u} & =\underline{0} & & \text { on } \partial \Omega-\Sigma_{\exp } \\
E C_{0} \varepsilon(\underline{u}) \underline{e}_{z} & =\underline{0} & & \text { on } \Sigma_{\exp }
\end{aligned}
$$

where $\underline{\operatorname{div}}, \varepsilon(\underline{u}), g$ and $\underline{e}_{z}$ are the divergence operator, the small strain tensor, the acceleration of gravity and an upward vertical vector respectively. $C_{0}$ is the normalized constitutive relation tensor, such that the stress tensor stands as $\sigma=E C_{0} \varepsilon(\underline{u})=E(\bar{\lambda}(\operatorname{tr} \varepsilon) I+2 \bar{\mu} \varepsilon)$, where $\bar{\lambda}=\frac{\nu}{(1-2 \nu)(1+\nu)}$ and $\bar{\mu}=\frac{1}{2(1+\nu)}$ are the normalized Lame coefficients, while $I$ and tr denote the identity tensor and the trace operator respectively. $\nu$ and $\rho$ stand for the Poisson's ratio and the mass density respectively, whose spatial distributions are assumed known a priori.

The previous group of equations can be rewritten in terms of a weak formulation as well. Given a Young's modulus spatial field $E$ belonging to a specific Hilbert space $\mathcal{P}$, one seeks $\underline{u}$ in another Hilbert space $\mathcal{V}$ such that:

$$
\int_{\Omega} \operatorname{tr} E C_{0} \varepsilon(\underline{u}) \varepsilon(\underline{w}) \mathrm{d} \Omega+\int_{\Omega} \rho g \underline{e}_{z} \cdot \underline{w} \mathrm{~d} \Omega=0 \quad \forall \underline{w} \in \mathcal{V}_{0}
$$

with $\mathcal{V}_{0}=\left\{\underline{w} \in \mathcal{V} \mid \underline{w}=\underline{0}\right.$ on $\left.\partial \Omega-\Sigma_{\text {exp }}\right\}$.

\section{THE INVERSE PROBLEM}

We consider that the inverse problem consists in determining the Young's modulus spatial field $E$ resulting in a displacement field on $\Sigma_{\text {exp }}$ as close to the experimental displacements $\underline{u}_{\exp }$ as possible.

\subsection{Formulation of the inverse problem}

Classically, the solution of the inverse problem consists in minimizing a misfit function, which is a given norm evaluating on $\Sigma_{\exp }$ the discrepancy between the displacement field $\underline{u}(E)$ associated with the forward problem and the experimental displacement field $\underline{u}_{\text {exp }}$ :

$$
\mathcal{J}_{0}(E)=\frac{1}{2} \int_{\Sigma_{\text {exp }}}\left\|\underline{u}(E)-\underline{u}_{\exp }\right\|^{2} \mathrm{~d} \Sigma_{\text {exp }}
$$

which emphasizes the determination of a best fit between experimental and numerical data.

Like any other inverse problem, this problem is ill-posed: the associated solution is unstable and not unique, in particular when it consists of a spatially-variable field in $\Omega$. This actually means from the numerical point of view that we have to find a large amount of scalar values, each being associated with a node of the FE mesh, whereas very few experimental data are available (in this case: only on the surface of the ground). 
To circumvent this issue, so-called Tikhonov's regularization terms are usually added to the previous norm, for instance to bound the magnitude of the sought spatial field or of its spatial gradient:

$$
\mathcal{J}(E)=\frac{1}{2} \int_{\Sigma_{\exp }}\left\|\underline{u}(E)-\underline{u}_{\exp }\right\|^{2} \mathrm{~d} \Sigma_{\exp }+\int_{\Omega} \mathcal{R}(E, \underline{\nabla} E) \mathrm{d} \Omega
$$

where $\underline{\nabla} E$ stands for the spatial gradient of $E$. We will discuss the choice of such regularization terms below.

\subsection{Adjoint state formulation}

The minimization of the previous misfit function (4) is usually performed by means of gradient-based techniques. In order to avoid time-consuming calculations as well as inaccuracies associated with numerical differentiation, the derivative of $\mathcal{J}(E)$ is analytically introduced by means of an adjoint state problem.

The solution $\underline{z}$ of this adjoint state problem can be considered as a Lagrange multiplier introduced in the following Lagrangian function $\mathcal{L}(\underline{u}, E, \underline{z})$ :

$$
\begin{aligned}
\mathcal{L}(\underline{u}, E, \underline{z})= & \frac{1}{2} \int_{\Sigma_{\text {exp }}}\left\|\underline{u}-\underline{u}_{\exp }\right\|^{2} \mathrm{~d} \Sigma_{\text {exp }}+\int_{\Omega} \mathcal{R}(E, \underline{\nabla} E) \mathrm{d} \Omega \\
& -\int_{\Omega} \operatorname{tr} E C_{0} \varepsilon(\underline{u}) \varepsilon(\underline{z}) \mathrm{d} \Omega-\int_{\Omega} \rho_{0} g \underline{e}_{z} \cdot \underline{z} \mathrm{~d} \Omega
\end{aligned}
$$

where $(\underline{u}, E, \underline{z})$ are considered independent. Minimizing $\mathcal{J}(E)$ with $\underline{u}$ verifying (1) is then equivalent to writing the first-order stationarity conditions for $\mathcal{L}(\underline{u}, E, \underline{z})$.

The first-order stationarity condition with respect to $\underline{u}$ leads to the adjoint state problem, which is very close to the forward problem:

$$
\int_{\Sigma_{\text {exp }}}\left(\underline{u}-\underline{u}_{e x p}\right) \cdot \underline{\delta u} \mathrm{~d} \Sigma_{\text {exp }}-\int_{\Omega} \operatorname{tr} E C_{0} \varepsilon(\underline{\delta u}) \varepsilon(\underline{z}) \mathrm{d} \Omega=0 \quad \forall \underline{\delta u} \in \mathcal{V}_{0}
$$

except for the associated loading conditions. Instead of body forces within the domain in (2), a boundary condition is applied to the surface of the ground $\Sigma_{e x p}$ and expressed as the discrepancy between the displacements $\underline{u}(E)$ and $\underline{u}_{e x p}$ :

$$
\begin{aligned}
\underline{\operatorname{div}} E C_{0} \varepsilon(\underline{z})=\underline{0} & \text { in } \Omega \\
\underline{z}=\underline{0} & \text { on } \partial \Omega-\Sigma_{\text {exp }} \\
E C_{0} \varepsilon(\underline{z}) \underline{n}=\underline{u}-\underline{u}_{\exp } & \text { on } \Sigma_{\text {exp }}
\end{aligned}
$$

Then the first-order derivative of $\mathcal{L}(\underline{u}, E, \underline{z})$ with respect to $E$ allows us to express the directional derivative of the misfit function easily as $D_{E} \mathcal{J}(E)=D_{E} \mathcal{L}(\underline{u}, E, \underline{z})$ with:

$$
\begin{aligned}
D_{E} \mathcal{L}(\underline{u}, E, \underline{z}) \delta E= & \int_{\Omega}\left(D_{E} \mathcal{R}(E, \underline{\nabla} E)-\operatorname{tr} C_{0} \varepsilon(\underline{u}) \varepsilon(\underline{z})\right) \delta E \mathrm{~d} \Omega \\
& +\int_{\Omega} D_{\underline{\nabla} E} \mathcal{R}(E, \underline{\nabla} E) \cdot \delta \underline{\nabla} E \mathrm{~d} \Omega \\
= & \int_{\Omega}\left(D_{E} \mathcal{R}(E, \underline{\nabla} E)-\operatorname{div} D_{\underline{\nabla} E} \mathcal{R}(E, \underline{\nabla} E)-\operatorname{tr} C_{0} \varepsilon(\underline{u}) \varepsilon(\underline{z})\right) \delta E \mathrm{~d} \Omega \\
& +\int_{\partial \Omega} D_{\underline{\nabla} E} \mathcal{R}(E, \underline{\nabla} E) \cdot \underline{n} \delta E \mathrm{~d} S
\end{aligned}
$$




\subsection{Numerical solution of the inverse problem}

The minimum of the misfit function $\mathcal{J}(E)$ is sought as $\nabla_{E} \mathcal{J}(E) \delta E=0 \forall \delta E$, which could be rewritten as the following optimality equations:

$$
\begin{aligned}
\operatorname{tr} C_{0} \varepsilon(\underline{u}(E)) \varepsilon(\underline{z}(E))=D_{E} \mathcal{R}(E, \underline{\nabla} E)-\operatorname{div} D_{\underline{\nabla} E} \mathcal{R}(E, \underline{\nabla} E) & \text { in } \Omega \\
D_{\underline{\nabla} E} \mathcal{R}(E, \underline{\nabla} E) \cdot \underline{n}=0 & \text { on } \partial \Omega
\end{aligned}
$$

The minimization problem eventually consists in solving three PDEs with unknowns $(\underline{u}(E), \underline{z}(E), E)$ : the forward problem (1), the adjoint problem (7) and the optimality equations (9). The identification process results in the solution of a system, which is highly nonlinear in the spatially-variable unknown field $E$.

The straightforward solution of this system first consists in choosing finite dimensional subspaces $\mathcal{V}_{h} \subset \mathcal{V}, \mathcal{V}_{0, h} \subset \mathcal{V}_{0}$ and $\mathcal{P}_{h} \subset \mathcal{P}$ using a typical Finite Element (FE) discretization with a given mesh $\mathcal{M}_{h}$. Then the discrete problem to solve reads:

$$
\begin{aligned}
& \int_{\Omega} \operatorname{tr} E_{h} C_{0} \varepsilon\left(\underline{u}_{h}\right) \varepsilon\left(\underline{w}_{h}\right) \mathrm{d} \Omega+\int_{\Omega} \rho g \underline{e}_{z} \cdot \underline{w}_{h} \mathrm{~d} \Omega=0 \quad \forall \underline{w}_{h} \in \mathcal{V}_{0, h} \\
& \int_{\Sigma_{e x p}}\left(\underline{u}_{h}-\underline{u}_{e x p}\right) \cdot \underline{w}_{h} \mathrm{~d} \Sigma_{e x p}-\int_{\Omega} \operatorname{tr} E_{h} C_{0} \varepsilon\left(\underline{z}_{h}\right) \varepsilon\left(\underline{w}_{h}\right) \mathrm{d} \Omega=0 \quad \forall \underline{w}_{h} \in \mathcal{V}_{0, h} \\
& \int_{\Omega}\left(D_{E_{h}} \mathcal{R}\left(E_{h}, \underline{\nabla} E_{h}\right)-\operatorname{tr} C_{0} \varepsilon\left(\underline{u}_{h}\right) \varepsilon\left(\underline{z}_{h}\right)\right) \delta E_{h} \mathrm{~d} \Omega \\
&+\int_{\Omega} D_{\underline{\nabla} E_{h}} \mathcal{R}\left(E_{h}, \underline{\nabla} E_{h}\right) \cdot \underline{\nabla} \delta E_{h} \mathrm{~d} \Omega \quad=0 \quad \forall \delta E_{h} \in \mathcal{P}_{h}
\end{aligned}
$$

with unknowns $\left(\underline{u}_{h}, \underline{z}_{h}, E_{h}\right) \in \mathcal{V}_{h} \times \mathcal{V}_{0, h} \times \mathcal{P}_{h}$.

In $[20,21]$, a complete investigation of how to solve this kind of inverse problem is proposed: the authors detailed the use of Newton-like methods using Krylov solutions at each Newton iteration. Here, the numerical method to solve the previous system (10) is a damped Newton method: at each step, the algorithm computes the analytical expression of the Jacobian matrix associated with the full system, then its numerical expression using the current values of the unknowns, and finds the best step length reducing the value of the residual associated with the system. The process is then stopped when the estimated relative error is below a given threshold. Other strategies could also be considered: instead of computing the Jacobian matrix associated with the full system, a staggered process could be proposed, where the forward and adjoint problems on the one hand, the optimality equation on the other hand, are solved successively.

\subsection{First identification results and shortcomings of the classical method}

For the time being, we use synthetic data as experimental data in order to study the influence of the regularization terms used in the inverse problem: the experimental data used here are actually simulated with a very fine 2D FE mesh $\mathcal{M}_{h h}$ based on a simplified reference model with only one homogeneous rectangular layer $\Omega$ whose mechanical properties are the following ones: $E_{0}=60 G P a$, $\nu_{0}=0.25, \rho_{0}=2,600 \mathrm{~kg} / \mathrm{m}^{3}$. The cavity is introduced as an elliptic homegeneous medium $\Omega_{\mathrm{c}}$ within $\Omega$, characterized by: $E_{\mathrm{c}}=0.6 G P a, \nu_{\mathrm{c}}=0.25, \rho_{\mathrm{c}}=2,600 \mathrm{~kg} / \mathrm{m}^{3}$. 
As a result, the so-called experimental displacements $\underline{u}_{e x p}$ are derived from the following problem:

$$
\begin{aligned}
& \int_{\Omega-\Omega_{\mathrm{c}}} \operatorname{tr} E_{0} C_{0} \varepsilon\left(\underline{u}_{\text {exp }}\right) \varepsilon\left(\underline{w}_{h h}\right) \mathrm{d} \Omega+\int_{\Omega-\Omega_{\mathrm{c}}} \rho_{0} g \underline{e}_{z} \cdot \underline{w}_{h h} \mathrm{~d} \Omega \\
& \quad+\int_{\Omega_{\mathrm{c}}} \operatorname{tr} E_{\mathrm{c}} C_{0} \varepsilon\left(\underline{u}_{e x p}\right) \varepsilon\left(\underline{w}_{h h}\right) \mathrm{d} \Omega+\int_{\Omega_{\mathrm{c}}} \rho_{\mathrm{c}} g \underline{e}_{z} \cdot \underline{w}_{h h} \mathrm{~d} \Omega=0 \quad \forall \underline{w}_{h} \in \mathcal{V}_{0, h h}
\end{aligned}
$$

Note that the fine mesh used here (about 50,000 quadratic triangular elements) to calculate $\underline{u}_{\exp }$ will not be used anymore in the following, for the solution of the inverse problem should not be dependent of the mesh used to solve the mechanical problem.

With this very simple example, we tested several regularization terms, using a mesh made of about 6,000 quadratic triangular elements. For each case, a homogeneous Young's modulus field equal to $E_{0}$ is used as an initial guess for the nonlinear solution of the system of equations (10), whereas the mass density is assumed to be equal to $\rho_{0}$. With this initial guess, the initial misfit function (with no regularization) $\mathcal{J}_{0}$ is about 5,600: let us recall that $\mathcal{J}_{0}$ is a straightforward indicator of the discrepancy between the experimental and the model-predicted displacements.

Choosing $\mathcal{R}(E, \underline{\nabla} E)=\frac{\alpha}{2}\left|E-E_{0}\right|^{2}$ means that the sought Young's modulus $E$ should remain close to the modulus $E_{0}$ of the homogeneous ground, which is known a priori. When spatially discretized, the associated optimality equation derived from (9) leads to the third equation of the system (10):

$$
\int_{\Omega}\left(\alpha\left(E_{h}-E_{0}\right)-\operatorname{tr} C_{0} \varepsilon\left(\underline{u}_{h}\right) \varepsilon\left(\underline{z}_{h}\right)\right) \delta E_{h} \mathrm{~d} \Omega=0 \quad \forall \delta E_{h} \in \mathcal{P}_{h}
$$

Of course, adding such a term results in a well-known, tricky choice of the constant $\alpha$. In Figure 2, the final value of the misfit function $\mathcal{J}_{0}$ obtained after solving the whole system (10) is plotted against the values of the regularization parameter $\alpha$. It can be seen that, if $\alpha$ is set too large, the homogeneous field $E_{0}$ is found as the solution and the misfit function $\mathcal{J}_{0}$ remains equal to its initial value (about 5,600), whereas if $\alpha$ is set too small, the regularization is insufficient to deal with the inverse problem and the algorithm fails to find the solution of the system (10). As a conclusion, it can be said that, whatever the choice of $\alpha$, it is quite impossible to solve the inverse problem and correctly identify the cavity without using another strategy.

Therefore we proceeded to the study of another regularization term, based on the gradient of the sought Young's modulus spatial field:

$$
\mathcal{R}(E, \underline{\nabla} E)=\frac{\beta}{2}\|\underline{\nabla} E\|^{2}
$$

which leads to the following discretized formulation:

$$
-\int_{\Omega} \operatorname{tr} C_{0} \varepsilon\left(\underline{u}_{h}\right) \varepsilon\left(\underline{z}_{h}\right) \delta E_{h} \mathrm{~d} \Omega+\int_{\Omega} \beta \underline{\nabla} E_{h} \cdot \underline{\nabla} \delta E_{h} \mathrm{~d} \Omega=0 \quad \forall \delta E_{h} \in \mathcal{P}_{h}
$$

This regularization seems more adapted to the considered inverse problem, because it binds the gradient of the sought field without restricting this latter close to a specific value. In order to choose a relevant value for the regularization parameter $\beta$, we proceed to the same analysis as for $\alpha$ : Figure 3 depicts the final values of the misfit function $\mathcal{J}_{0}$ after solving (10) for different values of $\beta$. For high values of $\beta$, the regularization is too restrictive and the homogeneous, initial field is found as the solution of the system (10). Then, when $\beta$ decreases, the final value of $\mathcal{J}_{0}$ decreases as well, until the algorithm fails to find the solution of (10), for values of $\beta$ lower than $6 \cdot 10^{-23}$. 


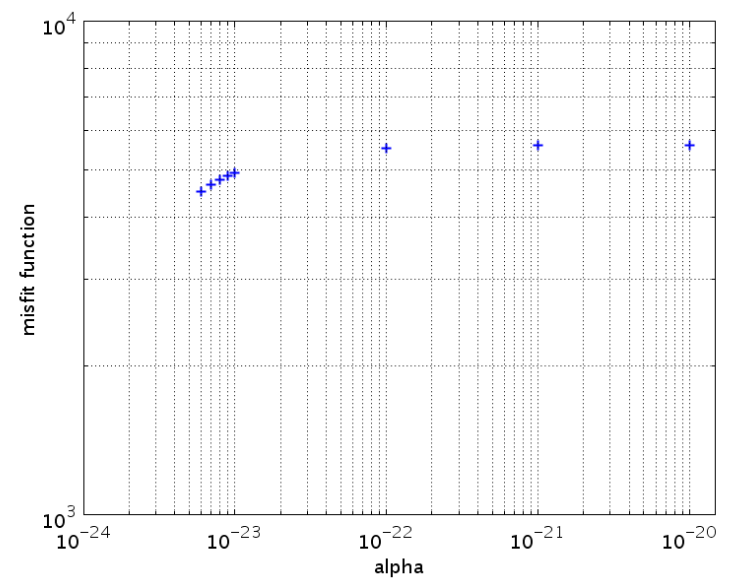

Figure 2. $\mathcal{J}_{0}$-vs.- $\alpha$ curve after solving (10): the algorithm fails to converge when $\alpha<6 \cdot 10^{-24}$

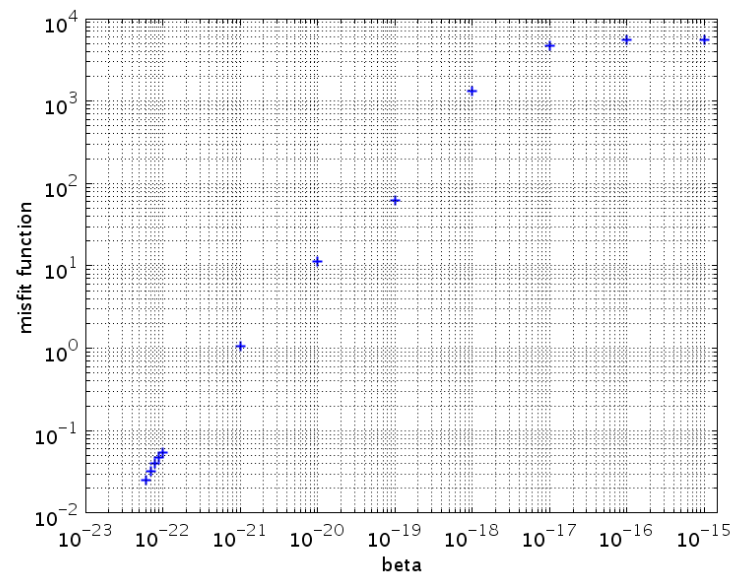

Figure 3. $\mathcal{J}_{0}$-vs. $-\beta$ curve after solving (10): the algorithm fails to converge when $\beta<6 \cdot 10^{-23}$

Nevertheless, finding a relevant value for $\beta$ is still a difficult process. For example, we can try to apply the Morozov discrepancy principle [22, 23], which consists in finding $\beta$ for which the final value of $\mathcal{J}_{0}$ after solving (10) is equal to the assumed accuracy or noise level. In the case of radar interferometry measurements, the accuracy can go up to a few millimiters, resulting to a final misfit function of about $10^{-1}$ to 1 in our proposed example. We see in Figure 3 that this level corresponds approximately to $\beta=10^{-21}$. But, actually, there may be additional noise, coming for example from the vegetal cover of the monitored ground, which makes the interferometric correlation coefficient drop significantly [19]. So, to check this choice, we added some Gaussian noise to the so-called experimental data $\underline{u}_{\text {exp }}$ : Figure 4 shows the values of the misfit function $\mathcal{J}_{0}$ after solving (10) for different values of 
$\beta$, when the standard deviation associated with the Gaussian noise is equal to $5 \mathrm{~cm}$. In this case, the level of the final misfit function should be about 25, and gives along with the Morozov discrepancy principle $\beta \approx 10^{-20}$.

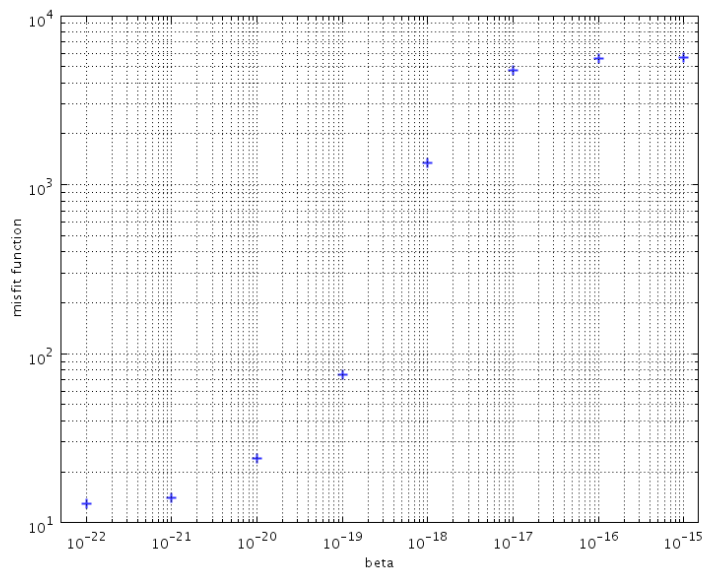

Figure 4. $\mathcal{J}_{0}$-vs. $-\beta$ curve after solving (10), with Gaussian noise added to $\underline{u}_{\text {exp }}$ : the algorithm fails to converge when $\beta<10^{-22}$

This previous case also shows that the noisier the data, the higher $\beta$ should be chosen to be able to solve the system (10). However, if $\beta$ is chosen larger, we get a solution with a very smoothed spatial variation, unable to represent the cavity that we introduced in (11) to synthesize the experimental data, as can be seen in Figure 5 where $\beta=10^{-19}$. The results of the identification are thus extremely dependent on the value of $\beta$.

A relevant choice of $\beta$ thus seems to be about $10^{-21}$ in the considered example. Another interpretation of this choice is that it should be made such that both terms in (4) would have the same magnitude: this could be achieved with:

$$
\beta \sim \frac{\left\|\underline{u}_{e x p}\right\|_{\infty}^{2}\left|\Sigma_{\exp }\right||\Delta x|^{2}}{\left|E_{0}\right|^{2}|\Omega|}
$$

where $|\Delta x|$ is an equivalent distance giving a rough estimate of the maximal spatial variation of the sought field. Here $\beta \approx 10^{-21}$ corresponds to $|\Delta x| \approx 25 \mathrm{~m}$.

As proposed in [24] for the same kind of inverse problem, one usual possibility to prevent the smoothing of the identified Young's modulus field is to choose the Total-Variation norm $\mathcal{R}(E, \underline{\nabla} E)=$ $\gamma\|\underline{\nabla} E\|$, or else its differentiable counterpart:

$$
\mathcal{R}(E, \underline{\nabla} E)=\gamma \sqrt{\|\underline{\nabla} E\|^{2}+\eta^{2}}
$$

which leads to the following discretized formulation:

$$
-\int_{\Omega} \operatorname{tr} C_{0} \varepsilon\left(\underline{u}_{h}\right) \varepsilon\left(\underline{z}_{h}\right) \delta E_{h} \mathrm{~d} \Omega+\int_{\Omega} \gamma \frac{\underline{\nabla} E_{h} \cdot \underline{\nabla} \delta E_{h}}{\sqrt{\|\underline{\nabla} E\|^{2}+\eta^{2}}} \mathrm{~d} \Omega=0 \quad \forall \delta E_{h} \in \mathcal{P}_{h}
$$

Such a choice tends to allow discontinuities in the identified field [25], which seems to be even more appropriate than the use of the gradient-based regularization with the L2-norm. 


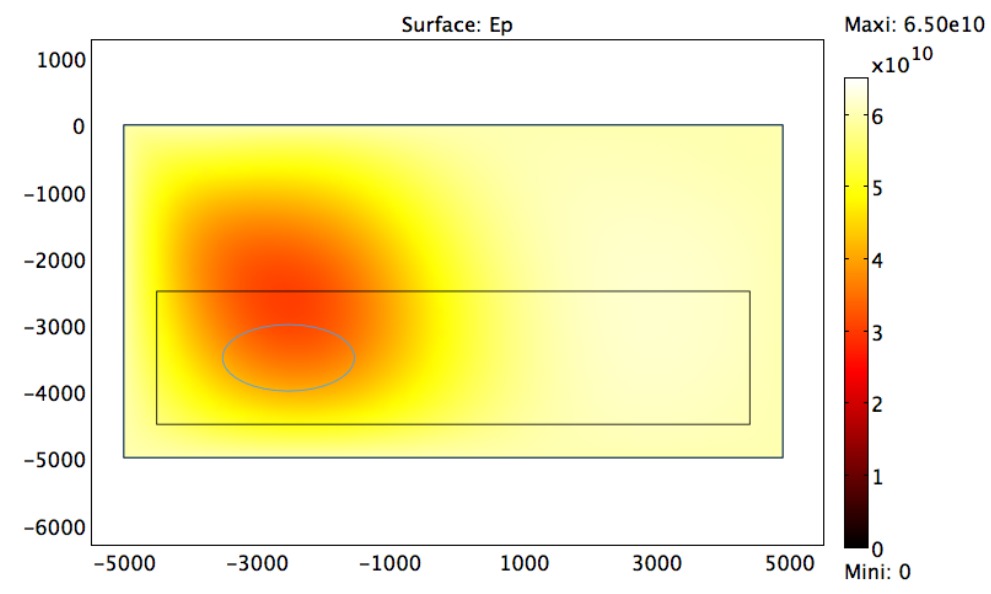

Figure 5. Shortcomings of the classical Tikhonov's regularization

The same analysis as before can be conducted, but now two different regularization parameters are to be set: $\gamma$ and $\eta$. The latter is classically chosen small, but first tests showed that in our case it had to be set far greater than expected to get the solution of the system (10): for $\eta^{2}<10^{6}$, it is impossible to find a solution different of the initial uniform field $E_{0}$ This is a first clue that the TV-based regularization can lead to numerical difficulties when solving (10). Figure 6 plots the misfit function after solving (10) against the values of $\gamma$, for $\eta^{2}$ set to $10^{9}$. The same curves are obtained when $\eta^{2}=10^{6}$ or $\eta^{2}=10^{12}$ are chosen. According to the Morozov discrepancy principle, for an accuracy of a few millimeters, $\gamma=10^{-14}$ could be chosen. Once again, to check this choice, we added some Gaussian noise to the socalled experimental data $\underline{u}_{e x p}$ : Figure 7 shows the values of the misfit function $\mathcal{J}_{0}$ after solving (10) for different values of $\gamma$, when the standard deviation associated with the Gaussian noise is equal to $5 \mathrm{~cm}$. In this case, we get $\gamma \approx 10^{-12}$. What is more significant is that the ability to solve the system (10) is strongly influenced by the noise level, far more than with gradient-based regularization with parameter $\beta$.

In what follows, we prefer to focus on the gradient-based regularization (13), because this choice seems less sensitive to the noise level. All in all, the regularization difficulties actually come from the fact that we want to determine a spatially-variable field represented by a large amount of scalar values to be identified, whereas experimental information is scarce. In addition to the noise, the mesh that is used for the discretization of this field influences the solution of the inverse problem. Indeed it is possible to show that with stronger assumptions reducing the number of sought parameters, the identification process is even more efficient. For example, if we know a rough estimate of the depth of the sought cavity, we can restrict the domain where the Young's modulus is sought for to a narrower area. Similarly, if we assume that the shape of the cavity is known, we can express the detection problem as the determination of the location and the size of the assumed shape. However, 


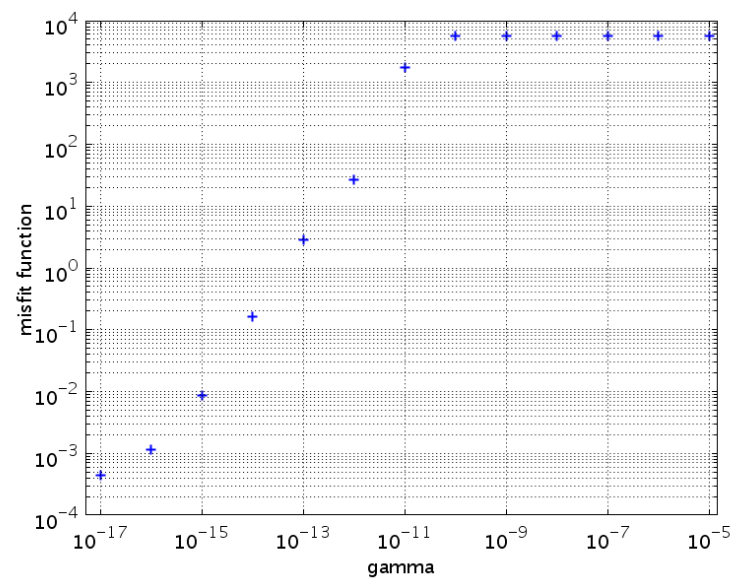

Figure 6. $\mathcal{J}_{0}$-vs.- $\gamma$ curve after solving (10): the algorithm fails to converge when $\gamma<10^{-17}$

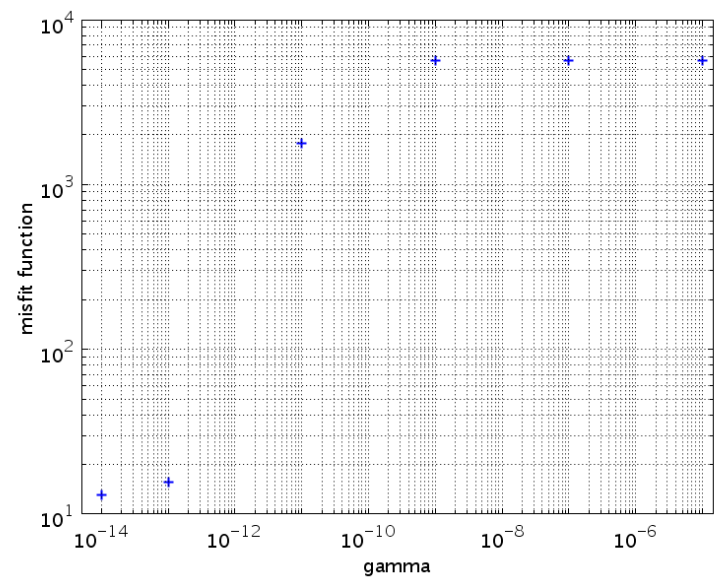

Figure 7. $\mathcal{J}_{0}$-vs.- $\gamma$ curve after solving (10), with Gaussian noise added to $\underline{u}_{\text {exp }}$ : the algorithm fails to converge when $\gamma<10^{-14}$

such assumptions are too restrictive, and make the robustness of the method drop dramatically, so they will not be considered in what follows. Here we prefer to focus on the discretization aspect of the inverse problem, to improve both the regularization of the inverse problem and the quality of the identified spatial field. 


\section{ITERATIVE STRATEGY USING MESH ADAPTION}

As we mentioned it before, the identification of the Young's modulus field is linked to the determination of the scalar unknowns associated with the discretization of this spatial field on a FE mesh. The coarser this mesh is, the fewer unknowns are to be found, and so the less ill-posed the inverse problem should be in terms of uniqueness and stability, for the unknown field is sought for in a smaller space. By this means, a slight reduction in the number of unknowns could be favourably obtained as well. However, using such a coarse mesh would not be convenient to deal with the solution of both forward and adjoint problems, if we wanted to get accurate results. Therefore we will need to go further in a second step to deal with this restrictive result, by introducing mesh adaptivity.

\subsection{Strategy using two different meshes}

The best trade-off is to introduce two distinct meshes: a sufficiently refined mesh $\mathcal{M}_{h}$ for the solution of the forward and adjoint problems (1) and (7) and a coarse mesh $\mathcal{M}_{H}$ for the discretization of the sought field $E$ and the solution of the optimality equation (9). Then the discrete problem consists in finding $\left(\underline{u}_{h}, \underline{z}_{h}, E_{H}\right) \in \mathcal{V}_{h} \times \mathcal{V}_{0, h} \times \mathcal{P}_{H}$ such that:

$$
\begin{aligned}
\int_{\Omega} \operatorname{tr} \Pi_{h}^{H} E_{H} C_{0} \varepsilon\left(\underline{u}_{h}\right) \varepsilon\left(\underline{w}_{h}\right) \mathrm{d} \Omega+\int_{\Omega} \rho_{0} g \underline{e}_{z} \cdot \underline{w}_{h} \mathrm{~d} \Omega & =0 \quad \forall \underline{w}_{h} \in \mathcal{V}_{0, h} \\
\int_{\Sigma_{\text {exp }}}\left(\underline{u}_{h}-\underline{u}_{e x p}\right) \cdot \underline{w}_{h} \mathrm{~d} \Sigma_{\text {exp }}-\int_{\Omega} \operatorname{tr} \Pi_{h}^{H} E_{H} C_{0} \varepsilon\left(\underline{z}_{h}\right) \varepsilon\left(\underline{w}_{h}\right) \mathrm{d} \Omega & =0 \quad \forall \underline{w}_{h} \in \mathcal{V}_{0, h} \\
\int_{\Omega}\left(D_{E_{H}} \mathcal{R}\left(E_{H}, \underline{\nabla} E_{H}\right)-\operatorname{tr} C_{0} \Pi_{H}^{h} \varepsilon\left(\underline{u}_{h}\right) \Pi_{H}^{h} \varepsilon\left(\underline{z}_{h}\right)\right) \delta E_{H} \mathrm{~d} \Omega & \\
+\int_{\Omega} D_{\underline{\nabla} E_{H}} \mathcal{R}\left(E_{H}, \underline{\nabla} E_{H}\right) \cdot \underline{\nabla} \delta E_{H} \mathrm{~d} \Omega & =0 \quad \forall \delta E_{H} \in \mathcal{P}_{H}
\end{aligned}
$$

where $\mathcal{V}_{h}$ and $\mathcal{P}_{H}$ are associated with the fine mesh $\mathcal{M}_{h}$ and the coarse mesh $\mathcal{M}_{H}$ respectively, and $\Pi_{H}^{h}: \mathcal{V}_{h} \rightarrow \mathcal{V}_{H}$ and $\Pi_{h}^{H}: \mathcal{P}_{H} \rightarrow \mathcal{P}_{h}$ are specific operators associated with pointwise mappings (projection and extension respectively). The system (18) is solved in the same way as the system (10) was previously, by using a damped Newton method.

In the following, we use the fine and coarse meshes depicted in Figures 8 and 9 respectively. $\mathcal{M}_{h}$ is made of about 6,000 quadratic triangular elements whereas $\mathcal{M}_{H}$ only has 12 linear triangular elements. The shape of the cavity introduced in the very fine calculation (11) of $\underline{u}_{e x p}$ is added for information purposes, but is not included in the identification process.

With such meshes, the solution of (18) using a gradient-based regularization term (13) with $\beta=10^{-21}$ leads to a good guess of the location of the sought cavity, which shows that it is possible to detect and approximately locate the cavity. The Young's modulus field, however, is identified in a very approximate way: Figure 10 shows the identified Young's modulus obtained with the two meshes described in Figures 8 and 9, as well as the shape of the elliptic cavity to be found. This first example thus shows that it is possible to detect the cavity, even if its shape cannot be precisely determined.

To quantify the quality of the identified Young's modulus, we define the following error indicator, which stands for the root mean square of the relative discrepancy between the experimental and forward displacement fields on the surface of the ground:

$$
e_{\Sigma_{e x p}}=\sqrt{\frac{\int_{\Sigma_{e x p}}\left\|\underline{u}-\underline{u}_{e x p}\right\|^{2} \mathrm{~d} \Sigma_{e x p}}{\int_{\Sigma_{e x p}}\left\|\underline{u}_{e x p}\right\|^{2} \mathrm{~d} \Sigma_{e x p}}}
$$




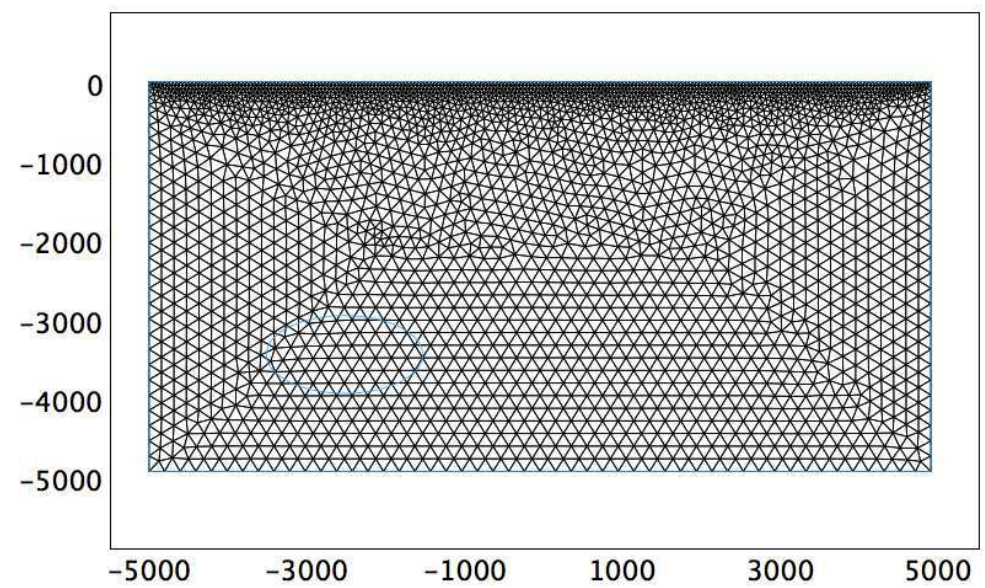

Figure 8. Example of a fine mesh $\mathcal{M}_{h}$ for the solution of forward and adjoint problems (5,758 quadratic triangular elements)

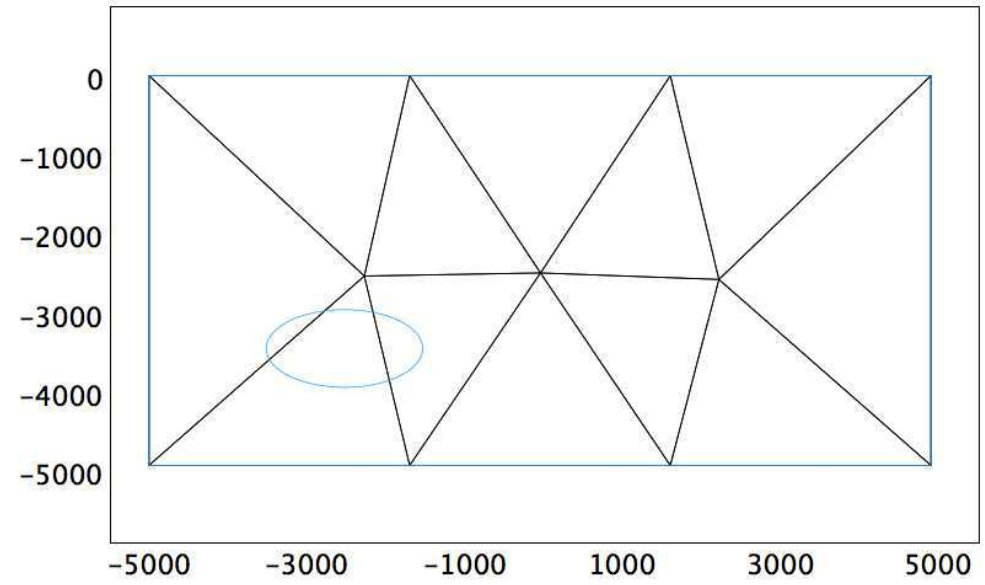

Figure 9. Example of an extremeley coarse mesh $\mathcal{M}_{H}$ for the discretization of the sought spatial field (12 linear triangular elements) 


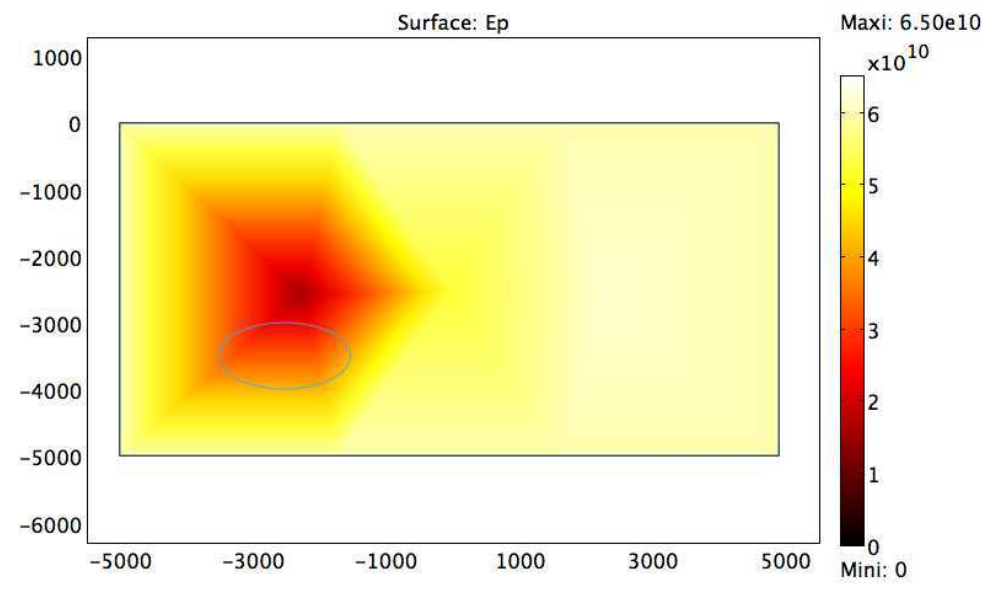

Figure 10. Young's modulus identified with meshes of Figures 8 and $9\left(e_{\Sigma_{e x p}}=1.37 \%\right)$

With the uniform Young's modulus $E_{0}$ as an initial guess, the previously defined error indicator reads $e_{\Sigma_{e x p}}=19.0 \%$. After the identification process, and despite the coarse description of the identified field, the error is quite low, even for an example with synthetic data: $e_{\Sigma_{e x p}}=1.37 \%$. A look at the two terms of the misfit function (4) after the identification process gives $\mathcal{J}_{0}=15$ and $\int_{\Omega} \frac{\beta}{2}\|\underline{\nabla} E\|^{2} \mathrm{~d} \Omega=3.0$ : the two terms are not perfectly balanced, the regularization term being weaker than the discrepancy term, but it was predictable because the coarse mesh used for the discretization of the identified field tends to have a regularizing effect.

To demonstrate this regularizing effect, we have plotted in Figure 11 the final values of the misfit function $\mathcal{J}_{0}$ after solving (18) for different values of $\beta$. When compared to Figure 3, the identification process using a coarse mesh $\mathcal{M}_{H}$ for the field $E_{H}$ is much more regularized, for values up to $10^{-40}$ are possible for the regularization parameter $\beta$. Of course, it is also observed that the solution is not improved any more below a given value of $\beta$, which roughly corresponds to the value obtained with (15).

\subsection{Iterative strategy with adaptive meshes}

To improve the identification further, we propose an iterative method based on Bangerth's work [14, 15]: the mesh $\mathcal{M}_{H}$ used for the discretization of the spatial field $E$ is progressively refined according to classical mesh adaption methods. These latter rely on a posteriori error estimators, such as estimators quantifying the quality of a mesh regarding the reference continuous mechanical problem. For implementation purposes, we begin to choose a L2-norm error indicator based on the residual $r$ 


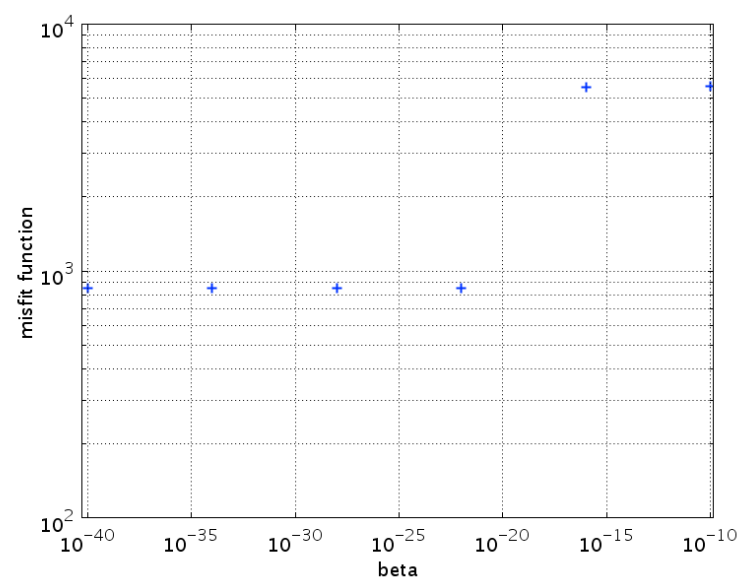

Figure 11. $\mathcal{J}_{0}$-vs. $-\beta$ curve after solving (18): the algorithm still converges when $\beta=\cdot 10^{-40}$

associated with equation (9):

$$
e_{L 2}=\sqrt{\int_{\Omega} H^{4}|r|^{2} \mathrm{~d} \Omega}
$$

where $H$ is the local size of the mesh $\mathcal{M}_{H}$. This choice can also be justified by the assumption that there is a constant $C$ such that the error $e$ verifies $|e| \leq C H^{2}|r|$. To evaluate the residual $r$, the solution of equation (9) is mapped on a mesh identical to $\mathcal{M}_{H}$, but with quadratic shape functions [26]. The local contribution for a given element is computed by averaging over the element the corresponding nodal values of $r$. The elements whose local contributions are the highest ones are refined: the algorithm selects these elements in a way that at each refinement step $k+1$, about three quarters of the initial number of elements of $\mathcal{M}_{H}^{k}$ are added to the mesh $\mathcal{M}_{H}^{k+1}$ used in the next step. The adaption steps stop when the global error is below a given threshold characterizing the quality of the solution of the optimality equation (9), and consequently of the identified spatial field $E$, or when no further improvement of the global error indicator is noticeable. Let us note that the idea of using for this kind of inverse problem a multiscale approach to enhance the chance of keeping the successive iterates within the basin of attraction of the global minimum was also proposed in [27], but this multigrid approach was not based on adaptive mesh refinement techniques.

Of course, it would be possible to use similar L2-norm error indicators to refine the mesh $\mathcal{M}_{h}$ as well. Here, however, this choice is not made for implementation purposes, and we assume that the mesh $\mathcal{M}_{h}$ is sufficiently refined for the solution of the forward and adjoint problems (1) and (7).

\subsection{Application of the strategy to the $2 D$ example}

This iterative strategy is applied to the problem of locating subterranean cavities using the synthetic data $\underline{u}_{e x p}$ derived from (11). Figure 12 illustrates the identification of an elliptic cavity using 6 adaption steps refining the initial discretization mesh $\mathcal{M}_{H}$ (12 linear elements) to a mesh $\mathcal{M}_{H}^{6}$ with about 2,000 elements in the end (Figure 13). The shape of the introduced cavity in (11) can be seen in both figures in the bottom left of the studied domain. 


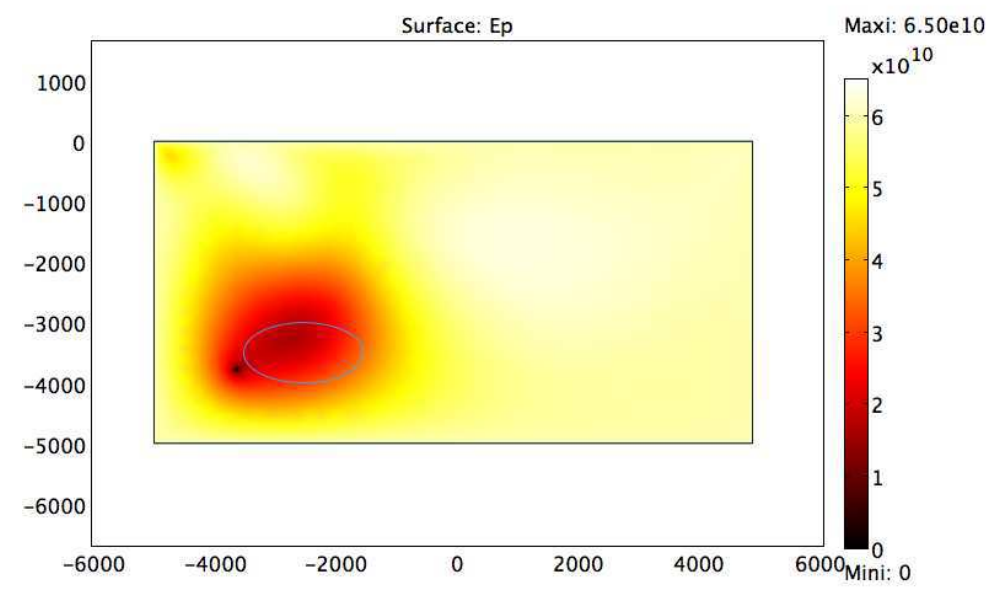

Figure 12. Young's modulus identified after 6 refinement steps $\left(e_{\Sigma_{e x p}}=0.235 \%\right)$

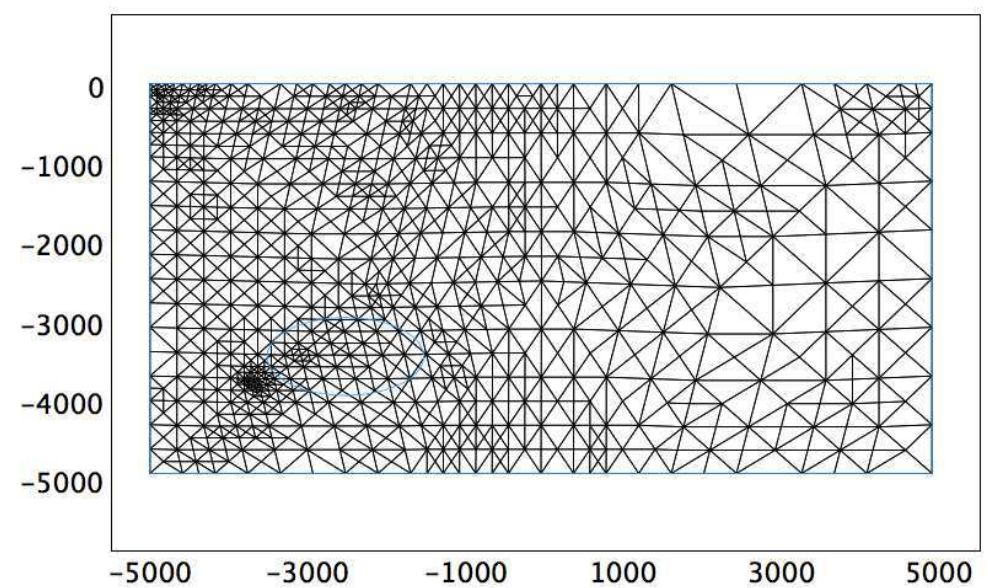

Figure 13. Discretization mesh $\mathcal{M}_{H}^{6}$ for the identified field after 6 refinement steps (1,758 linear triangular elements)

Figure 13 also demonstrates that the successive refinements occur near the location of the sought 
cavity, as well as close to the surface of the ground, where the solution of the adjoint problem is mainly located and influences the optimality equation (9). After these 6 iterations, the relative discrepancy indicator is $e_{\Sigma_{e x p}}=0.235 \%$, which shows the improvement of the identification by means of the iterative strategy (compared to $1.37 \%$ when using the mesh $\mathcal{M}_{H}$ and no refinement). Further refinements do not lead to any noticeable improvement. After these 6 refinement steps, the balance between the two terms of the misfit function (4) is the following: $\mathcal{J}_{0}=0.43$ and $\int_{\Omega} \frac{\beta}{2}\|\underline{\nabla} E\|^{2} \mathrm{~d} \Omega=4.6$. The discrepancy decreased while the regularization terms remained quite the same as previously, leading to a solution slightly oversmoothed.

\subsection{Influence of measurement noise}

The same strategy is then applied to the same example as in the previous paragraph, but to test the sensitivity of the method to noise, a Gaussian noise with a purposely overestimated $50 \mathrm{~cm}$-standard deviation is added to the experimental displacements $\underline{u}_{\text {exp }}$ derived from (11). The initial error indicator is $e_{\Sigma_{e x p}}=21.5 \%$. The identified Young's modulus is depicted in Figure 14: 6 iterative refinements of the mesh associated with the Young's modulus field have been achieved, and the relative discrepancy indicator is $e_{\Sigma_{\text {exp }}}=9.07 \%$.

When the forward displacement is calculated on $\Sigma_{\text {exp }}$ using the identified Young's modulus, this displacement field is very close to the experimental one before adding the Gaussian noise (a 'corrected' error estimator, where the noise has been erased, would give $e_{\Sigma_{e x p}}=0.949 \%$ ), which shows that the identification process tends to filter the noise on the experimental data, even at this high noise level. Figure 15 shows how the initial mesh associated with the discretization of the Young's modulus evolved into the mesh $\mathcal{M}_{H}^{6}$ with about 2,000 elements: due to the noise on the experimental data, more elements were refined on the vicinity of the surface of the ground than in the previous case.

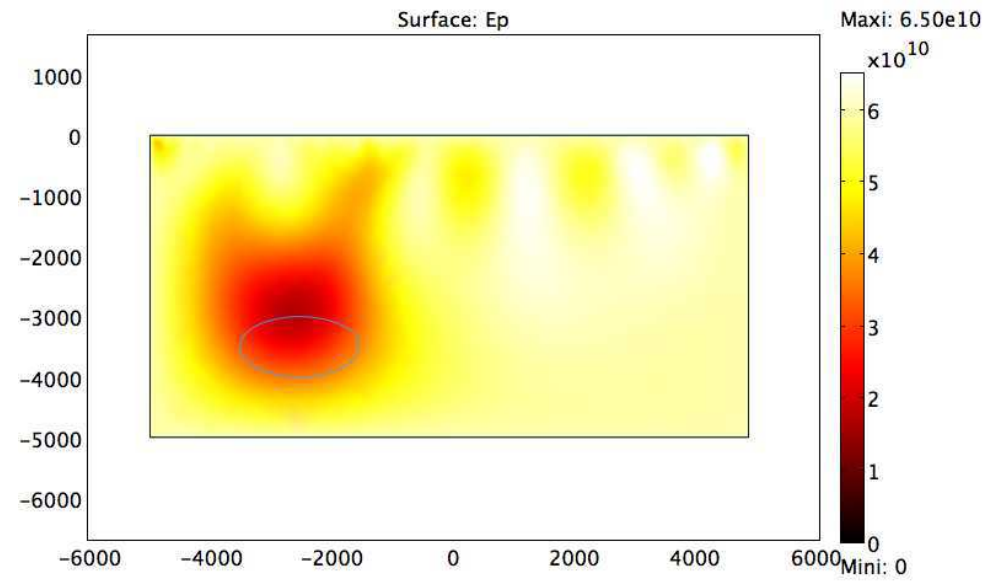

Figure 14. Young's modulus identified after 6 refinement steps in the case of experimental data with noise $\left(e_{\Sigma_{e x p}}=9.07 \%\right)$ 


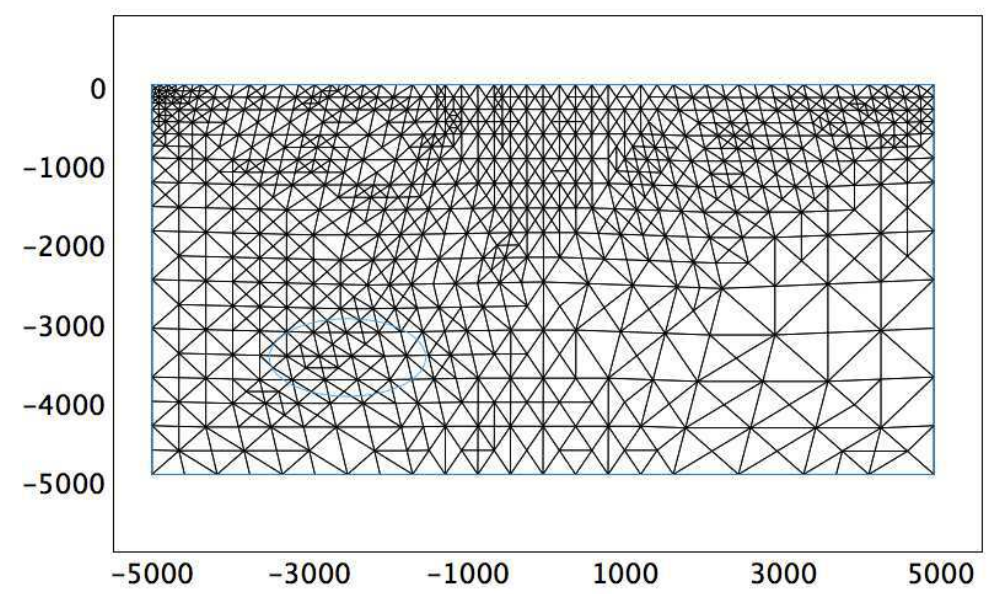

Figure 15. Discretization mesh $\mathcal{M}_{H}^{6}$ for the identified field after 6 refinement steps in the case of experimental data with noise (1,789 linear triangular elements)

This example tends to show the applicability of the method to actual interferometric radar measurements, for the strategy is able to deal with highly-corrupted data.

\subsection{Robustness of the strategy}

In order to demonstrate the robustness of the strategy, we create with a calculation analogous to (11) new synthetic displacements $\underline{u}_{\exp }$ associated with the case of two distinct subterranean cavities: the first one in the same location as in the previous examples, the second one in the vicinity of the surface of the ground. The initial error indicator with a uniform Young's modulus $E_{0}$ gives $e_{\Sigma_{e x p}}=25.7 \%$.

We apply then the iterative strategy beginning with the same two meshes $\mathcal{M}_{h}$ and $\mathcal{M}_{H}$ as in Figures 8 and 9 respectively. Figure 16 shows the identified Young's modulus after 5 refinement steps: when compared to the shapes indicating the locations of both cavities introduced in (11), this spatial field allows to determine clearly both cavities, even if the deepest one is only roughly located. Figure 17 depicts the associated mesh $\mathcal{M}_{H}^{6}$ (about 1,000 elements) after 6 refinement steps: the relative discrepancy indicator is $e_{\Sigma_{e x p}}=3.10 \%$, which is quite low (compared to $0.235 \%$ for the example with only one cavity). This example concludes on the quality of the identification and eventually shows the robustness of the introduced strategy: this is an additional point tending to demonstrate the applicability of the method to actual interferometric radar measurements.

\subsection{Application to a $3 D$ case}

Extending the strategy to the third dimension is straightforward. Experimental displacements are simulated with a very fine 3D mesh modeling the occurrence of a cavity in a calculation analogous to (11). As before, the iterative identification process is achieved using the experimental displacements $\underline{u}_{\text {exp }}$ on the surface of the ground. 


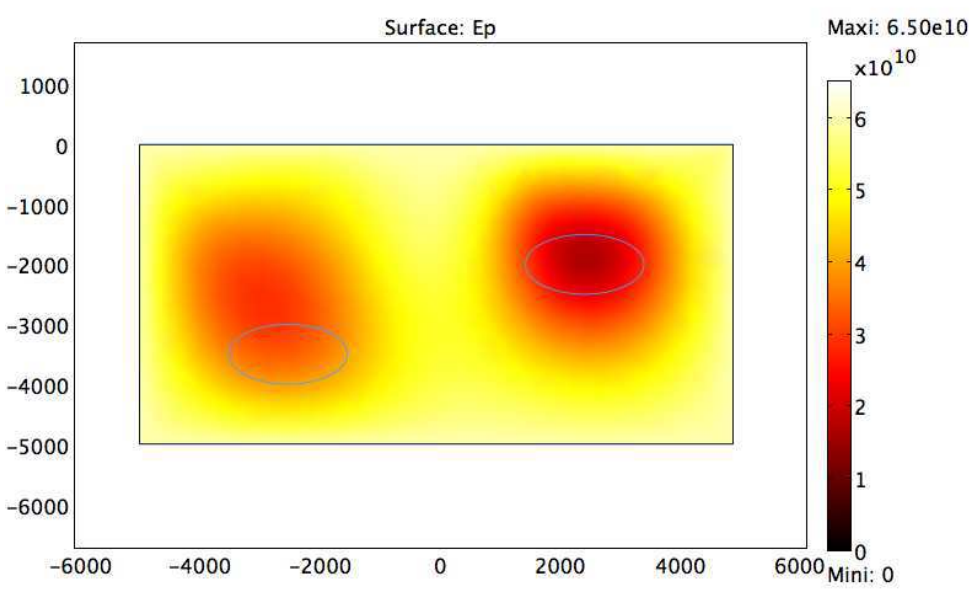

Figure 16. Young's modulus identified after 6 refinement steps in the case of two cavities $\left(e_{\Sigma_{e x p}}=3.10 \%\right)$

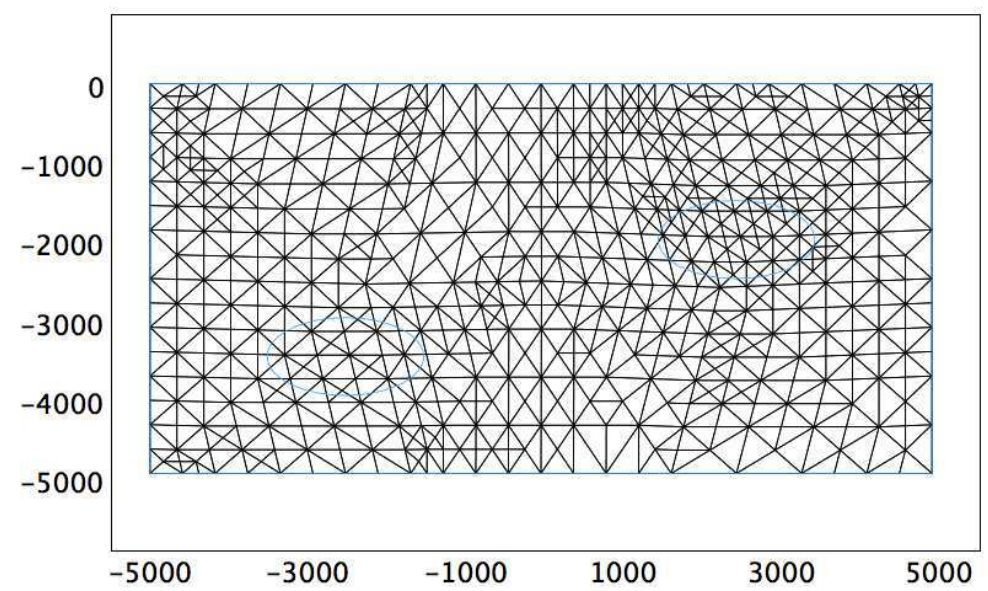

Figure 17. Discretization mesh $\mathcal{M}_{H}^{6}$ for the identified field after 6 refinement steps in the case of two cavities (1,148 linear triangular elements) 
Figure 18 shows 2D sections of the identified Young's modulus after 11 refinement steps. The coordinates of these $2 \mathrm{D}$ sections correspond to the coordinates of the center of the cavity used in (11) and its shape is drawn in the figure. In comparison with the shape of the sought cavity, we can see more clearly in Figure 19 each of these 2D sections, or in Figure 20 the $50 G P a$-isosurface. Eventually, the relative discrepancy indicator is $e_{\Sigma_{e x p}}=1.01 \%$, which is quite low and allows us to conclude on the quality of the identification in the $3 \mathrm{D}$ case.

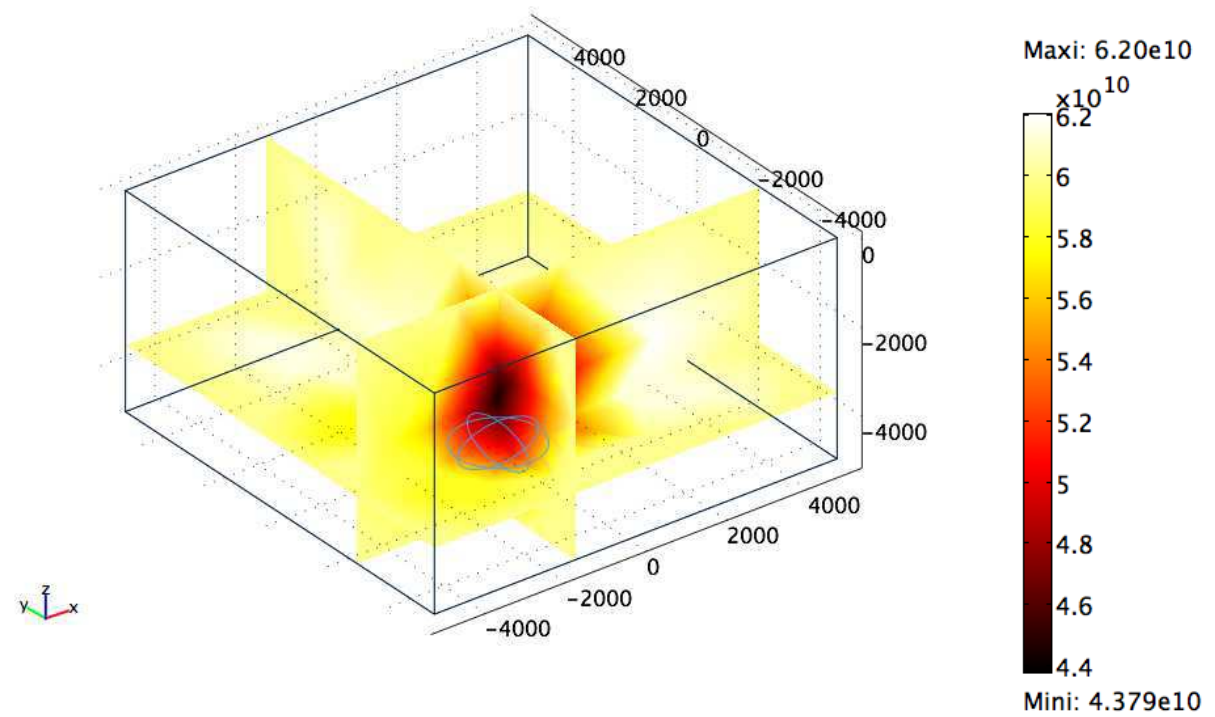

Figure 18. Young's modulus identified after 11 refinement steps in the 3D case $\left(e_{\Sigma_{e x p}}=1.01 \%\right)$

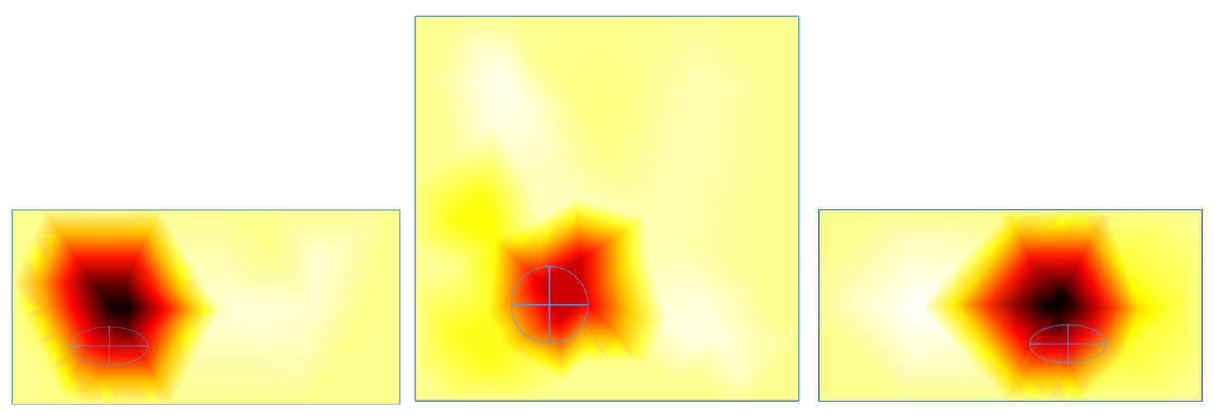

Figure 19. Young's modulus identified after 11 refinement steps in the 3D case - yz, xy and zx sections (from left to right) 


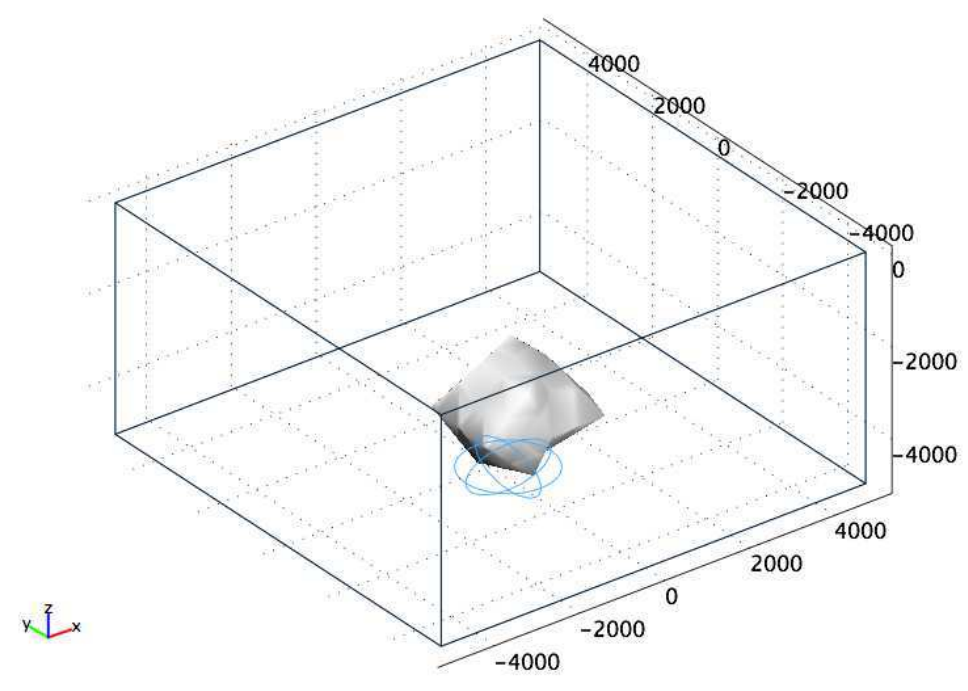

Figure 20. 50GPa-isosurface for the Young's modulus identified after 11 refinement steps in the 3D case

\section{FURTHER IMPROVEMENTS OF THE ITERATIVE STRATEGY}

From the previous section, we can conclude that the proposed strategy is both efficient and robust: for each case, it is possible to locate the sought cavity or cavities quite accurately. However, the Young's modulus spatial field that is finally identified is not the one that was used to simulate the experimental data $\underline{u}_{e x p}$ in (11). The main reason for this is that we used a regularization term based on the norm of the gradient: even if the proposed strategy relies on a progressively refined mesh $\mathcal{M}_{H}$, the identified field tends to be smoothed because the gradient-based regularization term (13) is minimized along with the initial misfit function (3). Although the uniqueness of the solution has been restored by this means, it is not possible to have a good guess of the size of the sought cavity.

\subsection{On the gradient-based regularization}

A possible solution would be to set different values of the gradient-based regularization term according to which step of the iterative strategy is concerned. Typical values such as (15) can be used in the first steps of the strategy, when regularization has to be sufficiently restrictive in order the identified field not to diverge, then lower values can be proposed in the last steps of the strategy, once the iterative mesh refinements concerning $\mathcal{M}_{H}$ drove the determination of the sought field to a given pattern. To do this, the following regularization term is proposed:

$$
\mathcal{R}(E, \underline{\nabla} E)=\frac{\beta(H)}{2}\|\underline{\nabla} E\|^{2}
$$


where $\beta$ is not a constant any more, but depends on the local size $H$ of the elements of $\mathcal{M}_{H}$ :

$$
\beta(H)=\frac{\left\|\underline{u}_{e x p}\right\|_{\infty}^{2}\left|\Sigma_{e x p}\right|}{\left|E_{0}\right|^{2}|\Omega|} H^{2}
$$

With such an expression, the regularization is strong when the element is large, its effect being reduced when the mesh $\mathcal{M}_{H}$ is refined. Typically, with such a regularization based on (22), the Young's modulus gradient should not be higher than that associated with a linear evolution of the Young's modulus along the element, that is $E_{0} / H$.

We used this evanescent gradient-based regularization with the previous 2D example (11). Figure 21 shows the identified Young's modulus after 6 iterations, while Figure 22 depicts the associated mesh $\mathcal{M}_{H}^{6}$ made of about 1,000 elements. Although the identified Young's modulus field seems to be smoothed once again, the relative discrepancy indicator is $e_{\Sigma_{e x p}}=0.170 \%$. We should recall that the relative discrepancy indicator associated with the initial gradient-based regularization (15) was $e_{\Sigma_{e x p}}=0.235 \%$ after 6 iterations.

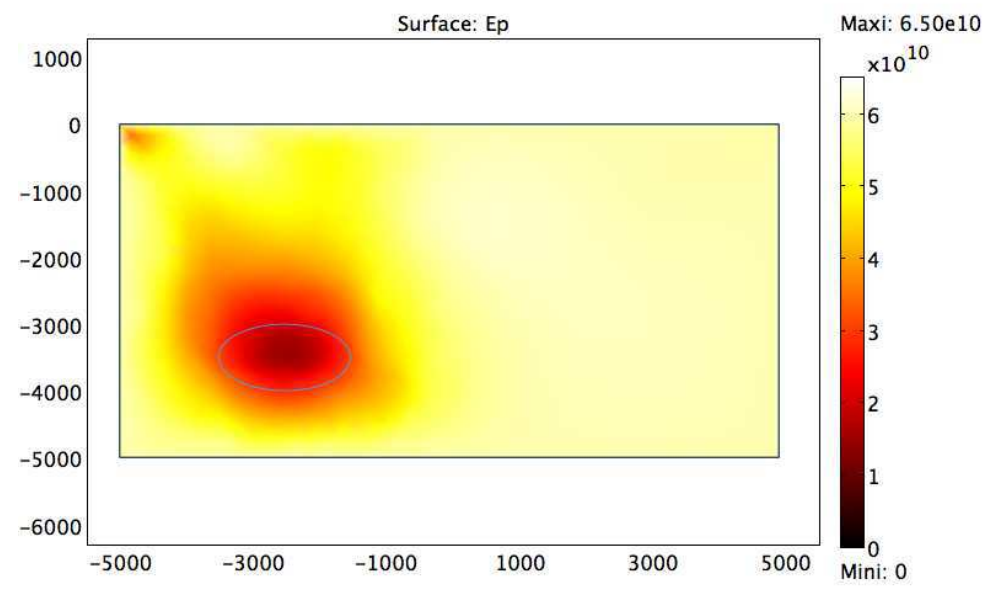

Figure 21. Young's modulus identified after 6 iterations with the evanescent gradient-based regularization $\left(e_{\Sigma_{e x p}}=0.170 \%\right)$

Attempts to propose a faster evanescent gradient-based regularization did not succeed, for the nonlinear algorithm to solve (18) did not converge any more after just a few steps.

\subsection{On the use of a quantity of interest}

Another limitation of the initial strategy lies in the fact that the closer we are to the minimum of the misfit function, the smaller the magnitude of the adjoint solution is, and so, the slower the convergence of the iterative strategy is, for the error estimator (20) driving the mesh refinement is associated with a vanishing optimality equation. In order to circumvent the evanescence of the adjoint solution, we can 


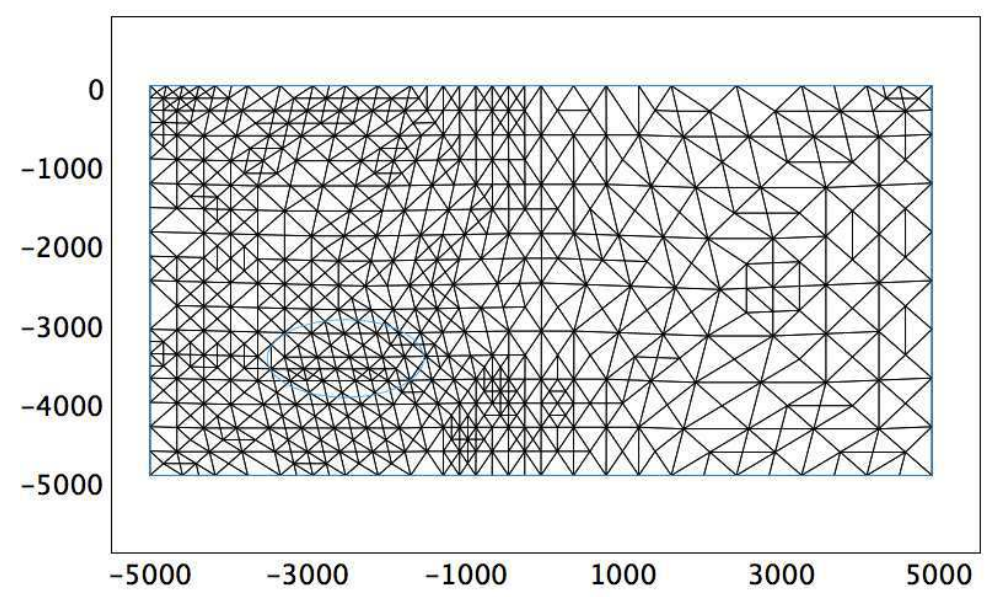

Figure 22. Discretization mesh $\mathcal{M}_{H}^{6}$ for the identified field after 6 iterations with the evanescent gradient-based regularization (1,353 linear triangular elements)

modify the initial Lagrangian function (5) (used to derive the stationarity conditions) by focusing on a so-called quantity of interest $\mathcal{E}(\underline{u}, E, \underline{z})$. The use of such a quantity has been proposed in $[16,17]$ to drive the mesh refinement in a parameter identification problem where the main target was the accurate estimation of the quantity of interest rather than the identification of the parameter field itself. In these references, only one mesh was used for discretizing the forward and adjoint problems along with the parameter field, and the mesh refinement was driven by error estimators related to the chosen quantity of interest. Here we are interested in extending this concept of quantity of interest to the case where two different meshes are used, even if the main target remains the identification of the Young's modulus spatial field. The idea is to drive the identification process with a more relevant mesh refinement than in the initial strategy, because, if appropriately chosen, the quantity of interest does not vanish in optimum, hence introducing a non-vanishing additional adjoint solution as well.

Here, as an example, we choose the following quantity of interest:

$$
\mathcal{E}(E)=\frac{1}{|\Omega|} \int_{\Omega} E \mathrm{~d} \Omega
$$

which represents the mean value of the Young's modulus spatial field over the whole domain $\Omega$. Such a quantity will not tend to zero as successive iterations are computed and can also be seen as a guess of the cavity's size: if it is assumed that the Young's modulus associated with the cavity is negligible when compared with that of the ground, the cavity's volume can be estimated with the following expression:

$$
\mathcal{V}_{c}=\left(1-\frac{\mathcal{E}(E)}{E_{0}}\right)|\Omega|
$$


The quantity of interest is introduced by means of a new Lagrangian function $\widehat{\mathcal{L}}(\underline{u}, E, \underline{z}, \underline{\hat{u}}, \hat{E}, \underline{\hat{z}})$ verifying:

$$
\begin{aligned}
\widehat{\mathcal{L}}(\underline{u}, E, \underline{z}, \underline{\hat{u}}, \hat{E}, \underline{\hat{z}})= & \mathcal{E}(\underline{u}, E, \underline{z}) \\
& +D_{\underline{u}} \mathcal{L}(\underline{u}, E, \underline{z}) \underline{\hat{u}}+D_{E} \mathcal{L}(\underline{u}, E, \underline{z}) \hat{E}+D_{\underline{z}} \mathcal{L}(\underline{u}, E, \underline{z}) \underline{\hat{z}}
\end{aligned}
$$

Whereas the first-order stationarity conditions relatively to the Lagrange multipliers $(\underline{\hat{u}}, \hat{E}, \underline{\hat{z}})$ lead to the initial group (1)-(7)-(9) of PDEs to be solved, three additional equations corresponding to the stationarity relatively to $(\underline{u}, E, \underline{z})$ can be derived:

$$
\begin{array}{cc}
-\int_{\Omega} \operatorname{tr} \hat{E} C_{0} \varepsilon(\underline{z}) \varepsilon(\underline{\delta u}) \mathrm{d} \Omega-\int_{\Omega} \operatorname{tr} E C_{0} \varepsilon(\underline{\hat{z}}) \varepsilon(\underline{\delta u}) \mathrm{d} \Omega & \\
+\int_{\Sigma_{e x p}} \underline{\hat{u}} \cdot \underline{\delta u} \mathrm{~d} S=-D_{\underline{u}} \mathcal{E}(\underline{u}, E, \underline{z}) \underline{\delta u} \quad \forall \underline{\delta u} \in \mathcal{V}_{0} \\
-\int_{\Omega} \operatorname{tr} \hat{E} C_{0} \varepsilon(\underline{u}) \varepsilon(\underline{\delta z}) \mathrm{d} \Omega-\int_{\Omega} \operatorname{tr} E C_{0} \varepsilon(\underline{\hat{u}}) \varepsilon(\underline{\delta z}) \mathrm{d} \Omega & \\
=-D_{\underline{z}} \mathcal{E}(\underline{u}, E, \underline{z}) \underline{\delta z} & \forall \underline{\delta z} \in \mathcal{V}_{0} \\
-\int_{\Omega} \operatorname{tr} \delta E C_{0} \varepsilon(\underline{\hat{u}}) \varepsilon(\underline{z}) \mathrm{d} \Omega-\int_{\Omega} \operatorname{tr} \delta E C_{0} \varepsilon(\underline{u}) \varepsilon(\underline{\hat{z}}) \mathrm{d} \Omega & \\
+\int_{\Omega} D_{E}^{2} \mathcal{R}(E, \underline{\nabla} E) \hat{E} \delta E \mathrm{~d} \Omega+\int_{\Omega} D_{\underline{\nabla} E}^{2} \mathcal{R}(E, \underline{\nabla} E) \underline{\nabla} \hat{E} \cdot \underline{\nabla} \delta E \mathrm{~d} \Omega & \\
=-D_{E} \mathcal{E}(\underline{u}, E, \underline{z}) \delta E & \forall \delta E \in \mathcal{P}
\end{array}
$$

These equations define a dual problem associated with the gradient of the quantity of interest. A close look at the equations of the previous system shows that the dual adjoint solution $\underline{\underline{z}}$ does not tend to zero as the primal forward solution $\underline{u}$ tends to the experimental data $\underline{u}_{\text {exp }}$.

Concerning the numerical solution of the dual problem, we use the same meshes as for the solution of the primal problem (1)-(7)-(9) : the fine mesh $\mathcal{M}_{h}$ for the solution of the equations associated with $\underline{\hat{u}}$ and $\underline{\hat{z}}$, and the coarse mesh $\mathcal{M}_{H}$ for the solution of the equation associated with $\hat{E}$. The numerical dual problem then consists in finding $\left(\underline{\hat{\hat{u}}}_{h}, \underline{\hat{\hat{z}}}_{h}, \hat{E}_{H}\right) \in \mathcal{V}_{0, h} \times \mathcal{V}_{0, h} \times \mathcal{P}_{H}$ such that:

$$
\begin{aligned}
& -\int_{\Omega} \operatorname{tr} \Pi_{h}^{H} \hat{E}_{H} C_{0} \varepsilon\left(\underline{u}_{h}\right) \varepsilon\left(\underline{w}_{h}\right) \mathrm{d} \Omega-\int_{\Omega} \operatorname{tr} \Pi_{h}^{H} E_{H} C_{0} \varepsilon\left(\underline{\hat{x}}_{h}\right) \varepsilon\left(\underline{w}_{h}\right) \mathrm{d} \Omega \\
& =-D_{\underline{z}_{h}} \mathcal{E}\left(\underline{u}_{h}, \Pi_{h}^{H} E_{H}, \underline{z}_{h}\right) \underline{w}_{h} \\
& -\int_{\Omega} \operatorname{tr} \Pi_{h}^{H} \hat{E}_{H} C_{0} \varepsilon\left(\underline{z}_{h}\right) \varepsilon\left(\underline{w}_{h}\right) \mathrm{d} \Omega-\int_{\Omega} \operatorname{tr} \Pi_{h}^{H} E_{H} C_{0} \varepsilon\left(\underline{\hat{z}}_{h}\right) \varepsilon\left(\underline{w}_{h}\right) \mathrm{d} \Omega \\
& +\int_{\Sigma_{\text {exp }}} \underline{\hat{u}}_{h} \cdot \underline{w}_{h} \mathrm{~d} S=-D_{\underline{u}_{h}} \mathcal{E}\left(\underline{u}_{h}, \Pi_{h}^{H} E_{H}, \underline{z}_{h}\right) \underline{w}_{h} \quad \forall \underline{w}_{h} \in \mathcal{V}_{0, h} \\
& -\int_{\Omega} \operatorname{tr} \delta E_{H} C_{0} \Pi_{H}^{h} \varepsilon\left(\underline{\hat{u}}_{h}\right) \Pi_{H}^{h} \varepsilon\left(\underline{z}_{h}\right) \mathrm{d} \Omega-\int_{\Omega} \operatorname{tr} \delta E_{H} C_{0} \Pi_{H}^{h} \varepsilon\left(\underline{u}_{h}\right) \Pi_{H}^{h} \varepsilon\left(\underline{\hat{z}}_{h}\right) \mathrm{d} \Omega \\
& +\int_{\Omega} D_{E_{H}}^{2} \mathcal{R}\left(E_{H}, \underline{\nabla} E_{H}\right) \hat{E}_{H} \delta E_{H} \mathrm{~d} \Omega+\int_{\Omega} D_{\underline{\nabla} E_{H}}^{2} \mathcal{R}\left(E_{H}, \underline{\nabla} E_{H}\right) \underline{\nabla} \hat{E}_{H} \cdot \underline{\nabla} \delta E_{H} \mathrm{~d} \Omega \\
& =-D_{E_{H}} \mathcal{E}\left(\Pi_{H}^{h} \underline{u}_{h}, E_{H}, \Pi_{H}^{h} \underline{z}_{h}\right) \delta E_{H} \\
& \forall \underline{w}_{h} \in \mathcal{V}_{0, h}
\end{aligned}
$$

where $\left(\underline{u}_{h}, \underline{z}_{h}, E_{h}\right) \in \mathcal{V}_{h} \times \mathcal{V}_{0, h} \times \mathcal{P}_{h}$ are derived from (18) as previously. Here, the successive refinements of $\mathcal{M}_{H}$ are driven by an error estimator analogous to (20), using the residual associated 
with the dual optimality equation (31), extending the study presented in [16, 17], where only one mesh was used, to the proposed strategy with two different meshes, where $\mathcal{M}_{H}$ is the specific mesh used for discretizing the sought spatial field.

We applied this primal-dual formulation with the quantity of interest (23) along with the evanescent gradient-based regularization (21) on the previous 2D example (11). Interesting is to note that we succeeded in setting a lower value for the evanescent regularization $\left(\beta(H) \approx 10^{-27} H^{2}\right)$ than the expression $(22)\left(\beta(H) \approx 10^{-26} H^{2}\right)$ that we used previously. The use of a quantity of interest seems to have a positive impact on the regularization of the inverse problem, for with lower values than $\beta(H) \approx 10^{-26} H^{2}$, there were problems of convergence in the solution of the initial problem associated with the system (18).

Figure 23 shows the identified Young's modulus after 6 iterations. Although the identified Young's modulus field seems to match less accurately the location of the cavity introduced in (11), the relative discrepancy indicator is $e_{\Sigma_{e x p}}=0.106 \%$, which is lower than with the previous evanescent gradientbased regularization (22) ( $e_{\Sigma_{e x p}}=0.170 \%$ after 6 iterations), and than with the initial gradient-based regularization (15) $\left(e_{\Sigma_{\text {exp }}}=0.235 \%\right.$ after 6 iterations).

The study of the quantity of interest is the following: after 6 iterations, $\mathcal{E}=54.5 G P a$, which corresponds to an estimated cavity's volume of about $\mathcal{V}_{c}=4.58 \mathrm{~km}^{3}$ (for a width of $1 \mathrm{~km}$ ). For the same width of considered ground, the actual volume of the cavity defined in (11) defining the experimental data was $\mathcal{V}_{c}=1.58 \mathrm{~km}^{3}$, since $\mathcal{E}=58.1 \mathrm{GPa}$. The estimated volume is then three times too high, which seems once again a consequence of choosing a gradient-based regularization. This conclusion, however, should be slightly nuanced, for the estimated cavity's volume with the previous formulations (for both initial gradient-based (15) and evanescent gradient-based regularization (22)) was $\mathcal{V}_{c}=5.25 \mathrm{~km}^{3}$ for a width of $1 \mathrm{~km}(\mathcal{E}=53.7 \mathrm{GPa})$. It seems then that using the average Young's modulus as quantity of interest led to a better estimation of the cavity's volume, even if this improvement remains restricted by the gradient-based regularization term.

Figure 24 depicts the associated mesh $\mathcal{M}_{H}^{6}$ made of about 300 elements only. The choice of a quantity of interest defined on the whole domain $\Omega$ seems to have driven a more homogeneous refinement of the mesh $\mathcal{M}_{H}$. Even if the finally obtained mesh $\mathcal{M}_{H}^{6}$ is still quite coarse, it is sufficient to describe the identified Young's modulus field in a better way than with the previous formulations, for $e_{\Sigma_{e x p}}=0.106 \%$ corresponds to the lowest value of the relative discrepancy indicator over all the identifications results after 6 iterations.

Even if these first conclusions need further investigations, using a quantity of interest to drive the mesh refinement on which the proposed strategy is based seems to improve both the regularization of the inverse problem and the result of the identification itself. In addition, the mesh $\mathcal{M}_{H}$ used for the discretization of the sought Young's modulus spatial field seems to have been refined in a more effective way, for the final error indicator is lower than with the other formulations, whereas the final mesh is coarser.

\section{CONCLUSION}

When dealing with the inverse problem of identifying a spatial field of material properties, one is confronted with the difficult choice of a relevant regularization. This is particularly true when the sought field is discretized on a FE mesh, for the choice of this latter can influence the result of the identification.

Here we introduce a general iterative strategy using adaptive meshes in order to circumvent the 


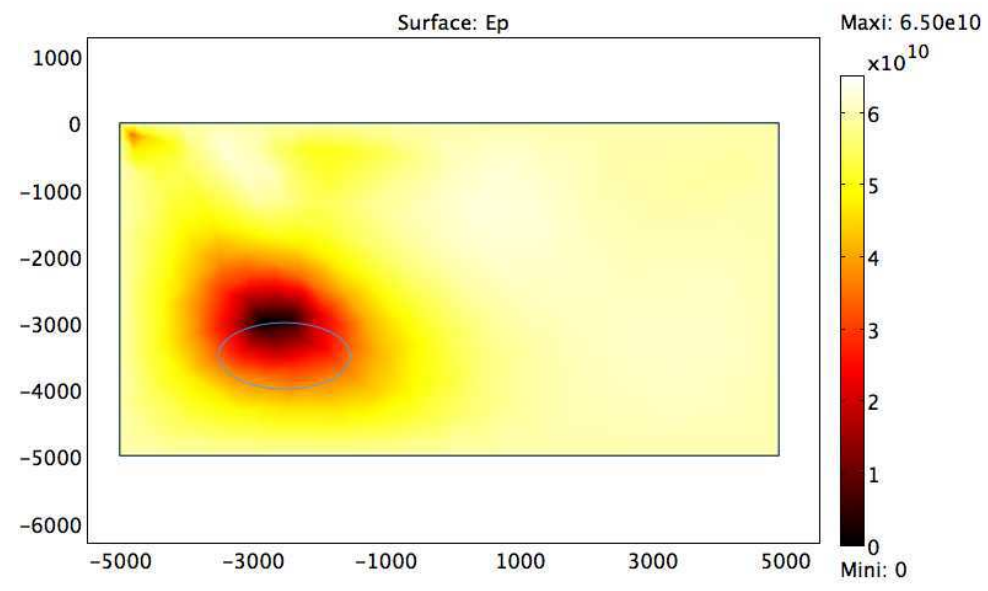

Figure 23. Young's modulus identified after 6 iterations: formulation with quantity of interest along with the evanescent gradient-based regularization $\left(e_{\Sigma_{\exp }}=0.106 \%\right)$

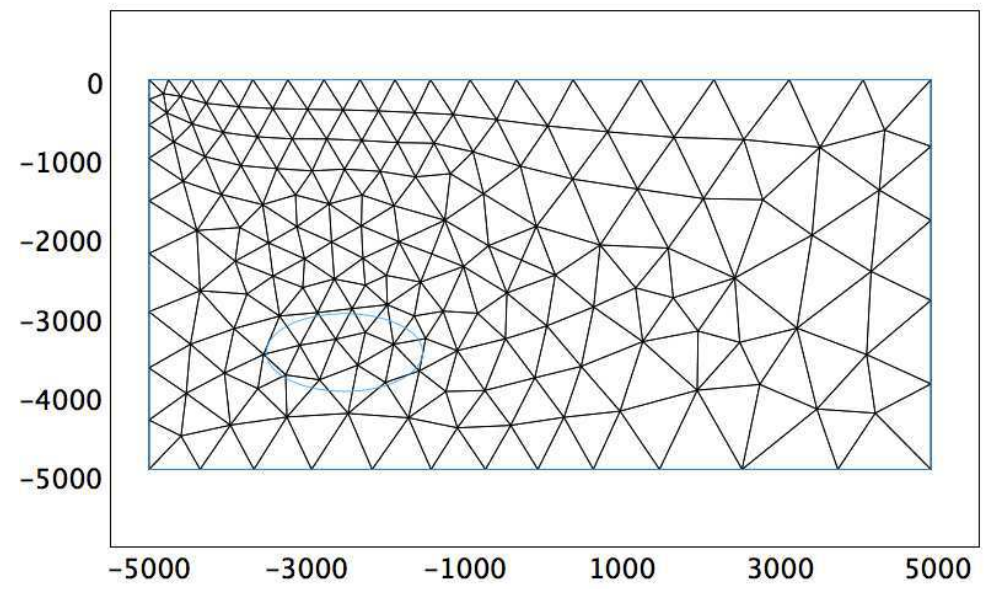

Figure 24. Discretization mesh $\mathcal{M}_{H}^{6}$ for the identified field after 6 iterations: formulation with quantity of interest along with the evanescent gradient-based regularization (305 linear triangular elements) 
shortcomings of the classical regularization methods. The goal is to use a specific mesh $\mathcal{M}_{H}$ for the spatial discretization of the field to be identified, independently from the mesh $\mathcal{M}_{h}$ used to discretize the forward and adjoint problems. The use of a coarse mesh for $\mathcal{M}_{H}$ makes the choice of the regularization terms easier, and the identification can be improved by refining the mesh $\mathcal{M}_{H}$ according to classical error estimators.

The robustness of such a strategy to solve spatial inverse problems has been illustrated in the specific case of the detection of underground cavities. In addition, several improvements of the initial formulation have been proposed so that this iterative strategy could give even more accurate identification results. The comparisons of the different formulations, however, are made difficult by the fact that the discrepancy indicator (19) that we introduced exhibits very low values in most cases, which may be associated with the synthetic data used. On the other hand, the addition of artificial noise to these synthetic data at a far higher level than the actual interferometric radar devices have tended to demonstrate the robustness of the strategy in the case of corrupted data. Therefore the successful application of this identification strategy on actual experimental data such as in [19] should be possible, and give in addition valuable information about the different choices made all along the strategy.

\section{REFERENCES}

1. Bonnet M, Constantinescu A. Inverse problems in elasticity. Inverse Problems 2005; 21(2):R1-R50.

2. Bonnet M. BIE and material differentiation applied to the formulation of obstacle inverse problems. Engineering Analysis with Boundary Elements 1995; 15(2):121-136.

3. Feijoo GR, Oberval A, Pinsky PM. An application of shape optimization in the solution of inverse acoustic scattering problems. Inverse Problems 2004; 20(1):199-228.

4. Burger M. Levenberg-Marquardt level set methods for inverse obstacle problems. Inverse Problems 2004; 20(1):259-282.

5. Constantinescu A. On the identification of elastic moduli from displacement-force boundary measurements. Inverse Problems in Engineering 1995; 1(4):293-315.

6. Feissel P, Allix O. Modified constitutive relation error identification strategy for transient dynamics with corrupted date: the elastic case. Computer Methods in Applied Mechanics and Engineering 2007; 196(13-16):1968-1983.

7. Ikehata M. Inversion formulas for the linearized problem for an inverse boundary value problem in elastic prospection. SIAM Journal in Applied Mathematics 1990; 50:1635-1644.

8. Nakamura G, Uhlmann G. Uniqueness for identifying Lamé moduli by Dirichlet to Neumann map. In Inverse problems in engineering sciences (ICM 90 Satellite Conference Proceedings), Yamaguti N. et al (eds). Springer Verlag, 1991.

9. Charambopoulos A, Gintides D, Kiriaki K. The linear sampling method for the transmission problem in three-dimensional linear elasticity. Inverse Problems 2002; 18(3):547-558.

10. Nintcheu Fata S, Guzina BB. A linear sampling method for near-field inverse problems in elastodynamics. Inverse Problems 2004; $20(3): 713-736$.

11. Bonnet M. Topological sensitivity for 3D elastodynamics and acoustic inverse scattering in the time domain. Computer Methods in Applied Mechanics and Engineering 2006; 195(37-40):5239-5254.

12. Ben Ameur H, Chavent G, Jaffré J. Refinement and coarsening indicators for adaptive parameterization: application to the estimation of hydraulic transmittivities. Inverse Problems 2002; 18:775-794.

13. Li R, Liu W, Ma H, Tang T. Adaptive finite element approximation for distributed elliptic optimal control problems. SIAM Journal in Control and Optimization 2002; 41:1321-1349.

14. Bangerth W. Adaptive Finite Elements Methods for the Identification of Distributed Parameters in Partial Differential Equations (PhD Thesis). University of Heidelberg: Germany, 2002.

15. Bangerth W, Joshi A. Adaptive finite element methods for the solution of inverse problems in optical tomography. Inverse Problems 2008; 24(3):034011.

16. Becker R, Vexler B. A posteriori error estimation for finite element discretization of parameter identification problems Numerische Mathematik 2003; 96(3):435-459.

17. Becker R, Vexler B. Mesh refinement and numerical sensitivity analysis for parameter calibration of partial differential equations. Journal of Computational Physics 2005; 206(1):95-110.

18. Ferretti A, Savio G, Barzaghi R, Borghi A, Musazzi S, Novali F, Prati C, Rocca F. Submillimeter accuracy of InSAR time series: experimental validation. IEEE Transactions on Geoscience and Remote Sensing 2007; 45(5):1142-1153.

19. Raucoules D, Maisons C, Carnec C, Le Mouelic S, King C, Hosford S. Monitoring of slow ground deformation by ERS 
radar interferometry on the Vauvert salt mine (France). Comparison with ground-based measurement. Remote Sensing of Environment 2003; 8:468-478.

20. Biros G, Ghattas O. Parallel Lagrange-Newton-Krylov-Schur methods for PDE-constrained optimization. Part I: the Krylov-Schur solver. SIAM Journal on Scientific Computing 2005; 27(2):687-713.

21. Biros G, Ghattas O. Parallel Lagrange-Newton-Krylov-Schur methods for PDE-constrained optimization. Part II: the Lagrange-Newton solver and its application to optimal control of steady flows. SIAM Journal on Scientific Computing 2005; 27(2):714-739.

22. Morozov V A. Methods for solving incorrectly posed problems. Springer: New York, 1984

23. Kaipio J, Somersalo E. Statistical and computational inverse problems. Springer: New York, 2005.

24. Gockenbach M, Khan A A. An abstract framework for elliptic inverse problems: Part 2. An augmented Lagrangian approach. Mathematics and Mechanics of Solids 2009; 14(6):517-539.

25. Acar R, Vogel C R. Analysis of bounded variation penalty methods for ill-posed problems. Inverse Problems 1994; 10(6):1217-1229.

26. Verfurth R. A review of a posteriori error estimation and adaptive mesh-refinement techniques. Wiley: Chichester, 1996.

27. Epanomeritakis I, Akcelik V, Ghattas O, Bielak J. A Newton-CG method for large-scale three-dimensional elastic fullwaveform seismic inversion. Inverse Problems 2008; 24:034015. 